\title{
Light Higgs Bosons in NMSSM at the LHC
}

\author{
MONORANJAn GUChaIT*1 AND JACKY KUMAR ${ }^{\dagger 2}$ \\ ${ }^{1,2}$ Department of High Energy Physics, Tata Institute of Fundamental Research, 400 005, Mumbai, India
}

\begin{abstract}
The next-to-minimal supersymmetric standard model (NMSSM) with an extended Higgs sector offers at least one Higgs boson as the Standard model (SM) like Higgs with a mass around $125 \mathrm{GeV}$. In this work, we revisit the mass spectrum and couplings of non-SM-like Higgs bosons taking into consideration most relevant constraints and identify the relevant parameter space. The discovery potential of these non-SM-like Higgs bosons, apart from their masses, is guided by their couplings with gauge bosons and fermions which are very much parameter space sensitive. We evaluate the rates of productions of these non-SM-like Higgs bosons at the LHC for a variety of decay channels in the allowed region of the parameter space. Although $b \bar{b}, \tau \tau$ modes appear to be the most promising decay channels, but for a substantial region of parameter space the two-photon decay mode has a remarkably large rate. In this study we emphasize that this diphoton mode can be exploited to find the non-SM-like Higgs bosons of the NMSSM and can also be a potential avenue to distinguish the NMSSM from the MSSM. In addition, we discuss briefly the various detectable signals of these non SM Higgs bosons at the LHC.
\end{abstract}

* guchait@.tifr.res.in

†jka@tifr.res.in 


\section{Introduction}

The recent discovery of the Higgs particle [1,2] has completed the particle family in the Standard model (SM), although more precise measurements are required to establish this claim strongly. On the other hand, there are various new physics models which can accommodate this Higgs candidate and are found to be consistent with the current measurements of Higgs couplings strength [3 3 . For example, the phenomenological models based on supersymmetric (SUSY) theory can interpret this Higgs candidate as their lightest Higgs boson 6, 7]. However, it is to be noted that to accommodate a Higgs boson of mass $\sim 125 \mathrm{GeV}$ in the theory of Minimal Supersymmetric Standard Model(MSSM), one requires a substantial contribution to the Higgs mass from higher order correction which is very close to the tree level value of it. Of course, this can be achieved in a very suitable region of SUSY parameter space, in particular with the contribution from the third generation squark sector along with some degrees of fine-tuning 8,9 .

The next-to-minimal supersymmetric standard model (NMSSM), an extension of MSSM with an additional Higgs singlet was proposed to solve the $\mu$ problem [10,11. Interestingly, it has been found that this model can provide a SM-like Higgs boson of mass around $125 \mathrm{GeV}$ more naturally (For example, see Refs. 12 20 and references therein), and as a result, after the discovery of the Higgs particle [1,2], it has received a lot of attention. In this model the additional interaction term between the singlet and the doublet Higgs superfields contributes substantially to the Higgs mass, even at the tree level and thus reduces the required fine tuning significantly. The phenomenology of the NMSSM, more precisely of its Higgs sector, is quite rich due to the presence of this extra Higgs singlet and the corresponding interaction with the Higgs doublet. In the literature, there exist several studies carried out to study the NMSSM Higgs sector in the context of the LHC. The recent observation of the Higgs particle at the LHC and related measurements of its properties renewed the interest in the NMSSM leading to more focused and dedicated activities. The implications of the current observation of the Higgs boson at the LHC and the measurements of its properties have been extensively discussed in the context of the NMSSM by many authors. In the LHC experiment looking for the NMSSM Higgs signal in the current Run I data sets is one of the ongoing studies. In a very recent analysis, very light Higgs boson in the NMSSM was studied and from the non-observation of any signal, a bound on the Higgs production cross section times the corresponding Higgs branching ratio (BR) is presented [21,22].

The NMSSM contains seven Higgs bosons, three are CP even Higgs scalars $\left(\mathrm{H}_{\mathrm{i}}, \mathrm{i}=1,2,3\right)$ and two are $\mathrm{CP}$ odd states, while there are two charged Higgs bosons. In this work we revisit the Higgs sector of the NMSSM by studying the interplay of model parameters and Higgs masses and couplings. We explore the possible implications in the NMSSM Higgs sector of recent Higgs discovery by scanning the model parameter space for a wide range requiring one of the CP even Higgs bosons SM-like Higgs with mass around $125 \mathrm{GeV}$. Performing a very comprehensive analysis taking into account various experimental and theoretical constraints, we delineate the region of parameter space which provides one of the $\mathrm{CP}$ even Higgs boson SM-like. Corresponding to this allowed parameter space, we then discuss the phenomenology of, particularly, light neutral non-SM-like CP even and odd Higgs bosons at the LHC. It is observed that there exist a variety of decay channels of these light neutral non SM Higgs bosons with very reasonable BR depending on the parameters points. In this work, we study very systematically in detail the sensitivity of these BRs on model parameters and their impact 
on light non-SM-like Higgs boson signals at the LHC. Moreover, in order to understand the feasibility of non-SM-like Higgs searches at the LHC, the rates of production of Higgs bosons in various decay channels are estimated for the center of mass energy $\sqrt{\mathrm{s}}=13 \mathrm{TeV}$, in the context of the interesting region of parameter space. In fact due to many parameters dependence of the cross sections and BRs of the NMSSM Higgs bosons, it is difficult to present systematically the variation of non SM Higgs boson production rates in various decay channels. Hence, we follow the strategy to present the ranges of these rates by computing the minimum and maximum values in various decay channels for a given Higgs boson mass and the parameter space. Naively, this estimation of rates provides hints about the detectability of non-SM-like Higgs bosons at the LHC although detail understanding of the background level is required to make a final conclusion. Interestingly, it is observed that the BR of nonSM-like Higgs in the di-photon channel is substantially large for a certain region of parameter space [18,23]. Undoubtedly, this two photons channel appears as a striking feature of the NMSSM model. Perhaps, the di-photon channel can provide a robust mode to distinguish the NMSSM from the MSSM because of the fact that photon is a clean object to probe experimentally. In addition there are also other hadronic decay channels of non SM Higgs bosons which can be useful as well in looking for Higgs signal in the NMSSM. It is to be noted that the similar type of study is carried out, in particular in Ref. 24 where the main focus is to study only the pair production of Higgs. In this paper, we study the production of Higgs via all dominant channels focusing the range of masses for all Higgs bosons below $125 \mathrm{GeV}$, the interesting range in the context of the present LHC experiments. In order to discuss the Higgs signal we have very systematically discussed the BR of various decay channels of non SM-like Higgs bosons including the two interesting channels, $g g$ and $c \bar{c}$. More importantly, in this current study we obtain rates for various decay channels estimating the Higgs boson production cross section following the model based calculation implemented in SuSHi 25].

The paper is organized as follows. In section 2 we discuss very briefly the relevant parts of the NMSSM Higgs sector. In section 3, describing inputs and constraints used while scanning parameters, we discuss the allowed ranges of the masses, the couplings and decay BRs of nonSM-like light Higgs bosons. Presenting the rates of the non-SM-like Higgs boson production in various channels in section 4, we discuss various Higgs signals in section 5. Finally we summarize our results in section 6 .

\section{NMSSM Higgs sector}

As mentioned in the introduction, the NMSSM Higgs sector contains one extra gauge singlet Higgs superfield $\hat{\mathrm{S}}$ in addition to two Higgs doublets $\left(\hat{\mathrm{H}}_{\mathrm{u}}, \hat{\mathrm{H}}_{\mathrm{d}}\right) \quad 1012$ making the super potential as,

$$
\mathrm{W}_{\mathrm{NMSSM}}=\mathrm{W}_{\mathrm{MSSM}}+\lambda \hat{S} \hat{\mathrm{H}}_{\mathrm{u}} \hat{\mathrm{H}}_{\mathrm{d}}+\frac{1}{3} \kappa \hat{\mathrm{S}^{3}},
$$

where $\mathrm{W}_{\text {MSSM }}$ corresponds to the super potential in MSSM with only two Higgs doublets without a $\mu$ term, $\lambda$ and $\kappa$ are dimensionless couplings. Recall that the primary goal in constructing the NMSSM is to generate the $\mu$ term dynamically to ensure its value around the EW scale, and thus solving the $\mu$ problem. The last term in Eq. 2.1 with $\hat{\mathrm{S}}^{3}$ is introduced to avoid Peccei-Quinn(PQ) symmetry [26]. Notice that this expansion respects explicit $Z_{3}$ symmetry. In the MSSM, $W_{\text {MSSM }}$ depends on $\hat{H}_{u}$ and $\hat{H}_{d}$, the Higgs super fields which couple with up-type quarks and down type quarks and charged leptons respectively. 
Correspondingly, the soft-SUSY breaking potential for NMSSM Higgs sector is given by,

$$
\mathrm{V}_{\text {soft }}=\mathrm{m}_{\mathrm{H}_{\mathrm{u}}}^{2}\left|\mathrm{H}_{\mathrm{u}}\right|^{2}+\mathrm{m}_{\mathrm{H}_{\mathrm{d}}}^{2}\left|\mathrm{H}_{\mathrm{d}}\right|^{2}+\mathrm{m}_{\mathrm{S}}^{2}|\mathrm{~S}|^{2}+\left[\lambda \mathrm{A}_{\lambda} \mathrm{SH}_{\mathrm{u}} \mathrm{H}_{\mathrm{d}}+\frac{1}{3} \kappa \mathrm{A}_{\kappa} \mathrm{S}^{3}+\text { h.c }\right] .
$$

Here $\mathrm{m}_{\mathrm{H}_{\mathrm{u}}}, \mathrm{m}_{\mathrm{H}_{\mathrm{d}}}, \mathrm{m}_{\mathrm{S}}, \mathrm{A}_{\lambda}$ and $A_{\kappa}$ are the soft breaking parameters. Notably, the parameters $\lambda, \kappa, \mathrm{A}_{\lambda}$ are taken to be positive. The vacuum expectation value (VEV) of the singlet field, $\langle\mathrm{S}\rangle=v_{s}$, in turn generates the effective $\mu$ term, $\mu_{\mathrm{eff}}=\lambda \mathrm{v}_{\mathrm{s}}$ and then restricting it at the order of EW scale. The neutral components of other Higgs doublets, get VEVs as $\left\langle\mathrm{H}_{\mathrm{u}}{ }^{0}\right\rangle=\mathrm{v}_{\mathrm{u}}$ and $\left\langle\mathrm{H}_{\mathrm{d}}{ }^{0}\right\rangle=\mathrm{v}_{\mathrm{d}}$ and the constraint $\mathrm{m}_{\mathrm{W}}^{2}=\frac{\mathrm{g}^{2}}{4}\left(\mathrm{v}_{\mathrm{u}}^{2}+\mathrm{v}_{\mathrm{d}}^{2}\right)$ makes one of the VEVs as a free parameter and it is parametrized as $\tan \beta=\mathrm{v}_{\mathrm{u}} / \mathrm{v}_{\mathrm{d}}$.

At the tree level, the Higgs sector of NMSSM have 9 parameters i.e,

$$
\lambda, \kappa, \tan \beta, \mu_{\mathrm{eff}}, \mathrm{A}_{\lambda}, \mathrm{A}_{\kappa}, m_{H_{u}}^{2}, m_{H_{d}}^{2}, m_{S}^{2} .
$$

But the minimization conditions of the scalar potential with respect to VEVs $v_{u}, v_{d}$ and $v_{s}$ reduce another three parameters, and hence, the NMSSM Higgs sector at the tree level is described by six independent parameters,

$$
\lambda, \kappa, \tan \beta, \mu_{\mathrm{eff}}, \mathrm{A}_{\lambda}, \mathrm{A}_{\kappa},
$$

unlike the MSSM where only two parameters $m_{A}$, the mass of the pseudoscalar Higgs and $\tan \beta$ are required. More detail discussions and review on the NMSSM Higgs sector can be found in Refs. $10-13,27,28]$.

The NMSSM Higgs sector contains 10 scalar degrees of freedom, out of which three are absorbed to give the masses of three gauge bosons, $\mathrm{W}^{ \pm}$and $\mathrm{Z}$ leading to seven physical Higgs states. Expansion of Higgs fields around the three vevs $v_{u}, v_{d}$ and $v_{s}$ yield various Higgs mixing terms in the Lagrangian of which real and imaginary parts constitute two $3 \times 3$ Higgs mass matrices corresponding to the CP-even and CP-odd scalars. The diagonalization of these mass matrices by orthogonal matrices provides masses of three physical Higgs bosons. For instance, the diagonalization of the $3 \times 3$ real mass matrix results in the masses of three $\mathrm{CP}$ even Higgs bosons $\left(\mathrm{H}_{1}, \mathrm{H}_{2}, \mathrm{H}_{3}\right)$ and the corresponding diagonalizing matrix relates the weak Higgs boson states, $\mathrm{H}_{\mathrm{j}}^{\mathrm{w}} \equiv\left(\mathrm{H}_{\mathrm{u}}{ }^{\mathrm{R}}, \mathrm{H}_{\mathrm{d}}{ }^{\mathrm{R}}, \mathrm{S}^{\mathrm{R}}\right)$ with the physical ones as,

$$
\mathrm{H}_{\mathrm{i}}=\mathrm{S}_{\mathrm{ij}} \mathrm{H}_{\mathrm{j}}^{\mathrm{w}} ; \quad \mathrm{i}, \mathrm{j}=1,2,3,
$$

where $\mathrm{S}_{\mathrm{ij}}$ is the orthogonal matrix diagonalizing the $3 \times 3 \mathrm{CP}$ even Higgs mass matrix. Here the $\mathrm{CP}$ even mass eigenstates $\left(\mathrm{H}_{\mathrm{i}}\right)$ arranged in increasing order of masses i.e $\mathrm{m}_{\mathrm{H}_{1}}<\mathrm{m}_{\mathrm{H}_{2}}<\mathrm{m}_{\mathrm{H}_{3}}$. Consequently, the physical Higgs boson states turn out to be admixtures of both doublet $\left(\mathrm{H}_{\mathrm{u}}, \mathrm{H}_{\mathrm{d}}\right)$ and singlet $(\mathrm{S})$ states and naturally the corresponding mixing angles affect their couplings to fermions and gauge bosons. Similarly, the imaginary parts of Higgs fields form a $3 \times 3$ mass matrix in the basis $\mathrm{A}_{\mathrm{j}}^{\mathrm{w}} \equiv\left(\mathrm{H}_{\mathrm{u}}^{\mathrm{I}}, \mathrm{H}_{\mathrm{d}}^{\mathrm{I}}, \mathrm{S}^{\mathrm{I}}\right)$ and is being diagonalized by an orthogonal matrix as,

$$
\mathrm{A}_{\mathrm{i}}=\mathrm{P}_{\mathrm{ij}} \mathrm{A}_{\mathrm{j}}^{\mathrm{w}}
$$

$A_{i}$ stands for three CP odd states, $\left(A_{1}, A_{2}, G^{0}\right)$, where $G^{0}$ is the Goldstone boson. Defining a suitable $2 \times 2$ matrix, the Goldstone boson state $\mathrm{G}^{0}$ can be rotated away leading only to two physical states $A_{1}$ and $A_{2}$ which are admixtures of doublet and singlet states determined by 
the components of the orthogonal matrix $\mathrm{P}_{\mathrm{ij}}$. The mixing angles i.e the matrix elements of the orthogonal matrices depend on the model parameters given by Eq,2.4 $[13,27,28$ and they play an important role in determining the couplings of the Higgs bosons with fermions and gauge bosons making those very much parameter space sensitive. For example, the couplings of $\mathrm{CP}$ even Higgs bosons $\mathrm{H}_{\mathrm{i}}$ to fermions, of both top and bottom type, are given by [13,27,28],

$$
\begin{gathered}
\mathrm{g}_{\mathrm{H}_{\mathrm{i}} \mathrm{t \overline { \textrm {t } }}}:-\frac{\mathrm{m}_{\mathrm{t}}}{\sqrt{2} \mathrm{v} \sin \beta} \mathrm{S}_{\mathrm{i} 1} ; \\
\mathrm{g}_{\mathrm{H}_{\mathrm{i}} \mathrm{b} \overline{\mathrm{b}}}: \frac{\mathrm{m}_{\mathrm{b}}}{\sqrt{2} \mathrm{v}_{\cos \beta}} \mathrm{S}_{\mathrm{i} 2} ; \\
\mathrm{g}_{\mathrm{H}_{\mathrm{i}} \tau \tau}: \frac{\mathrm{m}_{\tau}}{\sqrt{2} \mathrm{v} \cos \beta} \mathrm{S}_{\mathrm{i} 2},
\end{gathered}
$$

and similarly for CP odd scalars, $\mathrm{A}_{\mathrm{i}}$,

$$
\begin{gathered}
\mathrm{g}_{\mathrm{A}_{\mathrm{i}} \mathrm{t \overline {t }}}:-\mathrm{i} \frac{\mathrm{m}_{\mathrm{t}}}{\sqrt{2} \mathrm{v} \sin \beta} \mathrm{P}_{\mathrm{i} 2} ; \\
\mathrm{g}_{\mathrm{A}_{\mathrm{i}} \mathrm{bb}}: \mathrm{i} \frac{\mathrm{m}_{\mathrm{b}}}{\sqrt{2} \mathrm{v} \cos \beta} \mathrm{P}_{\mathrm{i} 1} ; \\
\mathrm{g}_{\mathrm{A}_{\mathrm{i}} \tau \tau}: \mathrm{i} \frac{\mathrm{m}_{\tau}}{\sqrt{2} \mathrm{v} \cos \beta} \mathrm{P}_{\mathrm{i} 1} .
\end{gathered}
$$

Here $\mathrm{S}_{\mathrm{i} 1}, \mathrm{~S}_{\mathrm{i} 2},\left(\mathrm{P}_{\mathrm{i} 1}, \mathrm{P}_{\mathrm{i} 2}\right)$ determine the amount of doublet composition in physical CP even(odd) Higgs boson states. Thus due to the presence of mixing angles, Higgs couplings either get suppressed or enhanced. For instance, if any of the Higgs state $\left(\mathrm{H}_{\mathrm{i}}, \mathrm{A}_{\mathrm{i}}\right)$ be dominated by singlet components i.e $\mathrm{S}_{\mathrm{i} 1}, \mathrm{~S}_{\mathrm{i} 2} \sim 0, \mathrm{P}_{\mathrm{i} 1}, \mathrm{P}_{\mathrm{i} 2} \sim 0$ then the corresponding Higgs boson couplings with fermions and gauge bosons are severely suppressed. The ratio between the Higgs couplings given in Eq. 2.7, 2.8 and its SM counter part is just a scale factor, called reduced coupling depending on mixing angle and $\tan \beta$, e.g $\mathrm{C}_{\mathrm{u}}=\mathrm{S}_{\mathrm{i} 1} / \sin \beta$ is the reduced coupling of $\mathrm{H}_{\mathrm{i}}$ with up-type quark. Moreover, the orthogonality property of the mixing matrices $(\mathrm{S}, \mathrm{P})$ predicts a sharing of couplings among the corresponding Higgs boson states. For example if any of the CP even Higgs boson be SM like i.e $\mathrm{S}_{\mathrm{i} 1}$ or $\mathrm{S}_{\mathrm{i} 2} \sim 1$, then the corresponding couplings of other $\mathrm{CP}$ even Higgs bosons with fermions are heavily suppressed. The coupling of $\mathrm{CP}$ even Higgs bosons with gauge bosons $(\mathrm{V}=\mathrm{W}, \mathrm{Z})$ are given by 13,28 ,

$$
\mathrm{g}_{\mathrm{H}_{\mathrm{i}} \mathrm{VV}}=\mathrm{g}_{\mathrm{H}_{\mathrm{i}}, \mathrm{VV}}^{\mathrm{SM}} \times \xi_{\mathrm{i}} ; \quad \xi_{\mathrm{i}}=\cos \beta \mathrm{S}_{\mathrm{i} 2}+\sin \beta \mathrm{S}_{\mathrm{i} 1},
$$

and because of orthogonality property of matrix S, one can show that the mixing angle follows the relation,

$$
\sum \xi_{\mathrm{i}}^{2}=1
$$

As stated earlier this sum rule implies that if any one of the Higgs boson couples dominantly with a gauge boson, then the same couplings of other Higgs bosons are severely disfavoured. The mixing angle $\xi_{\mathrm{i}}$ is constrained from the non observation of NMSSM Higgs bosons in LEP experiment 29].

In NMSSM the upper bound of lightest Higgs boson mass at tree level is well known to be as 10, 11, 27, 30, 31,

$$
\mathrm{m}_{\mathrm{H}_{1}}^{2} \lesssim \mathrm{M}_{\mathrm{Z}}^{2} \cos ^{2} 2 \beta+\lambda^{2} \mathrm{v}^{2} \sin ^{2} 2 \beta
$$


where the first term is similar to the upper bound $\left(=\mathrm{M}_{\mathrm{Z}} \cos 2 \beta\right)$ in MSSM and the second term originates due to the interaction between singlet and doublet Higgs field via $\lambda$ term, in Eq. 2.1. Remarkably, the contribution of the second term pushes this upper bound substantially much above $\mathrm{M}_{\mathrm{Z}} \cos 2 \beta$, of course, depending on the values of $\lambda$ and $\tan \beta$. Clearly, high (low) value of $\lambda(\tan \beta)$ is preferred to obtain a large value of the lightest Higgs mass at the tree level. Hence, this tree level NMSSM contribution to lightest Higgs mass enable to accommodate easily a SM-like Higgs mass of the range $\sim 125 \mathrm{GeV}$.

As pointed out earlier, the Higgs sector also receives higher order perturbative corrections contributed by squark masses and trilinear A-terms, and as well as by the parameters of the gaugino and Higgsino sectors. A very tiny contribution may come from slepton sectors also. The total extra contribution due to these corrections lead further enhancement of Higgs mass. The calculations of these higher order corrections exist in the literature $\mathcal{O}\left(\alpha_{\mathrm{t}} \alpha_{\mathrm{s}}+\alpha_{\mathrm{b}} \alpha_{\mathrm{s}}\right)[32$ 41] and the NMSSM-specific two loop corrections can also be found in the Ref. [40]. Including this additional correction to the mass, this upper limit of lightest Higgs boson mass can go up to $\sim 140 \mathrm{GeV}$ with the constraint that $\lambda$ to be within the perturbative limit 42,43 .

Consequently, the higher order correction to Higgs mass relates the other sectors, in particular third generation of squarks. Therefore to predict Higgs mass, one needs to take into consideration of involved model parameters, including very sensitive third generation sector. On the other hand, perhaps, the well determinations of Higgs sector predict about the range of masses and mixing angles of third generation squarks, namely top squarks [6, 44]. However, it is to be noted here that the different spectrum generators for the NMSSM predict different Higgs boson masses separated by few $\mathrm{GeV}$ for a given set of input parameters and schemes 45.

As described before, in NMSSM the Higgs sector has a non trivial dependence on a set of parameters Eq2.4 [28] which eventually affect the NMSSM Higgs phenomenology. Recall that generating $\mu$ term dynamically leads $\mu_{\text {eff }} \sim \lambda \mathrm{v}_{\mathrm{s}}$, which also is connected with chargino/neutralino masses. Hence, the LEP limit on light chargino mass $(\gtrsim 100 \mathrm{GeV})$ [46 predicts a bound, $\lambda \geq \mu_{\mathrm{eff}} / \mathrm{v}_{\mathrm{s}}$ [14]. Apparently, this bound restricts $\lambda$ not to be very small unless vev $v_{s}$ becomes too large, which is not also phenomenologically favourable as the other vevs, $v_{u}, v_{d}$ are expected to be also around the EW scale. However, there are certain variations of the NMSSM model, for instance, the semi-constrained NMSSM where soft masses of scalars, gauginos and trilinear A-terms for third generation fermions are assumed to be the same at the grand unified scale [47]. In this type of scenario, the values of the $\lambda$ and $\kappa$ can be very small, even much below of the $\mathcal{O}(0.1)$ which is completely compatible with the existing constraints which will be discussed later. On the other hand, running of $\lambda$ and $\kappa$ from high scale of Grand Unified Theory(GUT) to EW scale through renormalization group equation and the requirement of perturbative nature of couplings, a bound on them can be derived 28 as,

$$
\lambda^{2}+\kappa^{2} \lesssim 0.6
$$

\section{Scanning of NMSSM parameters}

In this section, we try to identify the region that offers one of the CP-even Higgs boson as the SM-like $\left(\mathrm{H}_{\mathrm{SM}}\right)$. by carrying out a comprehensive random scan of NMSSM parameter space. We use the package NMSSMTool4.3.0 [48,49] which calculates for a given set of input 
parameters, the masses, the couplings and the decay branching ratios of all the Higgs bosons along with the spectrum of other SUSY particles. This code also systematically checks the consistencies of the parameters against various theoretical as well experimental constraints. Experimental constraints include measurements of various low and high energy observables predicted within the framework of the NMSSM.

In our scan, we set the following ranges for the relevant parameters,

$$
\begin{aligned}
& 0.1<\lambda<0.7 ; \quad 0.1<\kappa<0.7 ; \quad 0<\mathrm{A}_{\lambda}<2 \mathrm{TeV},-2 \mathrm{TeV}<\mathrm{A}_{\kappa}<200 \mathrm{GeV} \\
& 1.5<\tan \beta<30 ; \quad 100 \mathrm{GeV}<\mu_{\mathrm{eff}}<2 \mathrm{TeV}
\end{aligned}
$$

Here the choice of parameters are phenomenologically motivated which expected to provide a very different scenario than the MSSM and testable at the future LHC experiments. The range of parameters are chosen in such a way that it provides one of the CP even Higgs boson to be SM-like with a mass around $125 \mathrm{GeV}$ and other singlet like Higgs bosons are not too massive. In our scan very small values of $\lambda$ are avoided to restrict $\mu_{e f f}$ and $\mathrm{v}_{\mathrm{s}}$ at the level of electroweak scale. However, as mentioned in sec.2, the values of $\lambda$ and $\kappa$ can be very small even much lower than our considered ranges, in the context of the certain variation of the NMSSM [47]. In view of this, our chosen scenario of the paratmeter choices is not very general one, represening only a subset of the parameter space. The g- 2 constraint favours the positive values of $\mu_{e f f}$. However, this in not a very general set up of parameters while more wider ranges of parameters are also accessible in the context of certain variation of NMSSM 47,50,51.

The soft mass parameters for the left and the right handed squarks from the first two generations, to which the Higgs boson mass are not so sensitive, are set to,

$$
\mathrm{M}_{\mathrm{Q}_{1 / 2}}=\mathrm{M}_{\mathrm{U}_{1 / 2}}=\mathrm{M}_{\mathrm{D}_{1 / 2 / 3}}=1 \mathrm{TeV},
$$

while for the third generation squarks, known to have a large effect on the Higgs mass, these are varied over the range,

$$
\mathrm{M}_{\mathrm{Q}_{3}}=\mathrm{M}_{\mathrm{U}_{3}}=300-3000 \mathrm{GeV} .
$$

to consider a wide range of values from them. However, as we know, because of radiative contributions to the mass of the Higgs boson, the masses of the top squarks and the corresponding mixing are important and hence A-term plays an important role. With a goal to achieve a CP even Higgs state close to the mass of the SM Higgs, we vary $\left|A_{t}\right|$ from small to large values as,

$$
\mathrm{A}_{\mathrm{t}}=-4 \mathrm{TeV} \text { to }+4 \mathrm{TeV},
$$

and setting other third generation trilinear A-terms as,

$$
\mathrm{A}_{\mathrm{b}}=2 \mathrm{TeV} ; \text { and } \mathrm{A}_{\mathrm{E}_{3}}=1 \mathrm{TeV},
$$

while keeping all other remaining trilinear A terms to zero. While scanning parameters, we test all constraints including theoretical ones pertaining to vacuum stability. Moreover, the precision measurements at the LEP experiments restrict the new physics models through the measurement of $\mathrm{M}_{\mathrm{W}}, \sin ^{2} \theta_{\mathrm{eff}}$, the $\rho$ parameter and also via the invisible decay width of the $\mathrm{Z}$ boson. In addition, the constraints due to various low energy observables from flavor physics 
such as $\mathrm{B} \rightarrow \mathrm{X}_{\mathrm{s}} \gamma, \mathrm{B}_{\mathrm{s}} \rightarrow \mu^{+} \mu^{-}$and $\mathrm{B} \rightarrow \tau^{+} \nu_{\tau}$ and also the mass differences $\Delta \mathrm{M}_{\mathrm{d}}$ and $\Delta \mathrm{M}_{\mathrm{s}}$ are checked in the process of scanning of the parameters. Various existing bounds on sparticle masses and production cross sections that are obtained from LEP, the Tevatron and from the LHC experiments are also imposed. The details of all these constraints can be found in 52. Notably, among various experimental constraints, the most crucial ones are from the Planck [53 data and the muon anomalous magnetic moment [54]. In this model the lightest neutralino $\left(\tilde{\chi}_{1}^{0}\right)$ is assumed to be the lightest-supersymmetric particle (LSP) and is also a DM candidate. NMSSMTools interfaced with micrOMEGAs [55,56], calculates the relic density. Recent data from the PLANCK experiment [53] concludes the relic density to be,

$$
\Omega^{2} \mathrm{~h}=0.1187 \pm 0.0017 .
$$

In the NMSSM, the DM solution is tested with the measured data as shown above at the $10 \%$ level. The most natural solution to the DM problem is via the bulk annihilation channel which requires lighter electroweak gauginos and sleptons. The impact of DM constraints on the NMSSM are discussed by many authors $[16,47,56,66$. The parameters in the gaugino sector, $\mathrm{M}_{1}$ and $\mathrm{M}_{2}$, the $\mathrm{U}(1)$ and the $\mathrm{SU}(2)$ gaugino masses, respectively are expected not to have any large effects on Higgs masses, but are important in calculating the dark mater relic density, Eq. 3.18. Therefore, we vary the values of gaugino masses $\mathrm{M}_{1}, \mathrm{M}_{2}$ while keeping $\mathrm{M}_{3}$, which is very close to the value of the gluino mass to a fixed value,

$$
50 \mathrm{GeV}<\mathrm{M}_{1}<1 \mathrm{TeV} ; \quad 50 \mathrm{GeV}<\mathrm{M}_{2}<1 \mathrm{TeV} ; \quad \mathrm{M}_{3}=1.2 \mathrm{TeV} .
$$

The precisely measured value of the muon anomalous magnetic moment,

$$
\mathrm{a}_{\mu}=\frac{\left(\mathrm{g}_{\mu}-2\right)}{2}
$$

is considered to be a very strong constraint for new physics. The total SM contribution to $\mathrm{a}_{\mu}$ is estimated with an uncertainty predominantly due to the hadronic contributions [67]. The discrepancy between the measured value and the one predicted by the SM is found to be [54],

$$
\delta \mathrm{a}_{\mu}=(28.7 \pm 8.0) \times 10^{-10} .
$$

This implies the measured value is $3 \sigma$ away from the SM predicted value. In SUSY model, there are additional contributions due to the presence of sparticles in loops which provide an explanation for this excess 68 71. In the NMSSM, the effect of $g_{\mu}-2$ constraint on its model parameter space has been discussed in [72. As we know, the main SUSY contribution to $\mathrm{a}_{\mu}$ comes at the one loop level involving smuon-neutralino and sneutrino(muon)-chargino diagrams. However, this SUSY contribution at the one loop level is determined by the sign of the $\mu$-term and the value of $\tan \beta$. In order to have a reasonable contribution to $\delta \mathrm{a}_{\mu}$ from these additional one loop SUSY diagrams, the masses of the sparticles, in particular of the smuons are favoured to be as light as $\sim 100 \mathrm{GeV}$ and $\tan \beta \sim 10-1572$. The slepton masses are not directly coupled with the Higgs sector, but plays a crucial role in determining the anomalous magnetic moment of the muon as discussed before. In order to have a parameter space consistent with $g_{\mu}-2$ constraint we assume low values of the first two generations of sleptons [72] i.e,

$$
\mathrm{M}_{\mathrm{L}_{1,2}}=100 \mathrm{GeV} ; \quad \mathrm{M}_{\mathrm{E}_{1,2}}=100 \mathrm{GeV} .
$$




\begin{tabular}{|l|ll|}
\hline & Lower & Upper \\
\hline$C_{t}$ & 0.97 & 2.28 \\
$C_{b}$ & 0.00 & 1.23 \\
$C_{V}$ & 0.66 & 1.23 \\
$C_{g}$ & 0.52 & 1.07 \\
$C_{\gamma}$ & 0.67 & 1.33 \\
\hline
\end{tabular}

Table 1: The allowed ranges of reduced couplings of the SM-like Higgs with fermions and the gauge bosons and also the effective couplings with the gluons and the photons at 95\%C.L. These are obtained by CMS experiments [3].

The Higgs contribution to $\delta \mathrm{a}_{\mu}$ comes via 2-loop diagrams 73,74$]$ and is found to be negligible both in the SM and in the MSSM because of the higher values of the Higgs masses. On contrary, in the NMSSM rather light Higgs states decoupled from fermions and bosons are viable, even with a few $\mathrm{GeV}$ mass. The one loop diagrams involving these lighter Higgs states are found to be potential sources contributing to $\delta \mathrm{a}_{\mu}[65,72]$. For instance, for lower values of the Higgs mass $\lesssim 10 \mathrm{GeV}$, for both $\mathrm{CP}$ even and $\mathrm{CP}$ odd states, contributions appear to be substantial within $2 \sigma$ of the central value of $\delta \mathrm{a}_{\mu}$ given in Eq. 3.21 for large values of $\tan \beta$. In our scan, we take into account the impact of $g_{\mu}-2$ constraint which has some effects on the parameter space as will be discussed later.

In the NMSSM, out of three CP even Higgs bosons, one is required to be SM-like. In addition, the couplings of the SM-like Higgs boson to fermions and the gauge bosons should be consistent with the current measurements by both the CMS and the ATLAS experiments [3]. As shown in Eq2.7, 2.8 and 2.9, the couplings of the Higgs boson with fermions and the gauge boson are basically a scaling of the corresponding SM couplings by a factor called the reduced couplings which are essentially the ratios of $\mathrm{g}_{\mathrm{H}_{\mathrm{i}} \mathrm{ff}} / \mathrm{g}_{\mathrm{H}_{\mathrm{i}} \mathrm{ff}}^{\mathrm{SM}}\left(=\mathrm{C}_{\mathrm{f}}\right)$ or $\mathrm{g}_{\mathrm{H}_{\mathrm{i}} \mathrm{VV}} / \mathrm{g}_{\mathrm{H}_{\mathrm{i}} \mathrm{VV}}^{\mathrm{SM}}\left(=\mathrm{C}_{\mathrm{V}}\right)$. Similarly, one can also have reduced effective couplings of Higgs with gluons and photon, say $\mathrm{C}_{\mathrm{g}}$ and $\mathrm{C}_{\gamma}$, respectively. At the LHC, both the ATLAS and the CMS collaborations examined the the Higgs couplings following the strategy discussed in Ref. 75]. By performing a very detailed analysis, the allowed ranges of the reduced couplings are reported at 95\% C.L. In Table 1 allowed ranges of these reduced couplings measured by the CMS experiment 3 are presented. Note that the CMS and ATLAS experiments do not measure the Higgs couplings directly. However, by measuring the signal strength, the factors by which the SM couplings are scaled can be constrained. Here these scale factors are defined to be the reduced couplings which are constrained by both the LHC experiments. It is to be noted that the SM Higgs production cross sections in various related modes are used in deriving these limits. In our study, while scanning the parameter space, we impose these constraints on the reduced couplings for the SM-like CP even Higgs boson. Similarly, from negative searches of Higgs at the LEP experiment, the reduced couplings of the SM-like Higgs boson with W/Z $\left(\mathrm{C}_{\mathrm{v}}=\xi\right)$ also get constrained [29] and our scan has been subjected to these constraints as well. Furthermore, in the NMSSM, possibly the SM-like Higgs boson can decay via non-standard modes which will be discussed in later section. The total BR in these non SM modes, namely $\mathrm{BR}_{\mathrm{BSM}}$ of the SM like Higgs boson is also restricted from above from the current Higgs data. The CMS measurements set the upper limit of non-SM branching ratio of the SM-like Higgs 
to be $[3$,

$$
\mathrm{BR}_{\mathrm{BSM}} \leq 0.57 \text { at } 95 \% \text {.C.L. }
$$

This limit is incorporated in our parameter scan.

Eventually, after playing with the NMSSM model parameters, we identify the region of parameter space allowed by all theoretical and experimental constraints as described.above, which yields one of the relatives light $\mathrm{CP}$ even Higgs bosons, either $\mathrm{H}_{1}$ or $\mathrm{H}_{2}$, to be SM-like with its mass in the range,

$$
\mathrm{m}_{\mathrm{H}_{1}} / \mathrm{m}_{\mathrm{H}_{2}} \sim \mathrm{m}_{\mathrm{H}_{\mathrm{SM}}}=125.02 \pm 3 \mathrm{GeV} .
$$

It is always observed that the heaviest $\mathrm{CP}$ even neutral Higgs, $\mathrm{H}_{3}$ tends to be massive $\sim 200$ $\mathrm{GeV}$ or more. Thus, we focus our attention on two distinct regions presenting the following two scenarios:

- Case A: the second lightest $\mathrm{CP}$ even Higgs, $\mathrm{H}_{2} \sim \mathrm{H}_{\mathrm{SM}}$.

- Case B: the lightest $\mathrm{CP}$ even state, $\mathrm{H}_{1} \sim \mathrm{H}_{\mathrm{SM}}$.

In the subsequent sections we discuss various features of these two cases and the relevant phenomenology in the context of ongoing LHC experiment with a center of mass energy of $13 \mathrm{TeV}$.

\subsection{Case A: $\mathrm{H}_{2} \sim \mathrm{H}_{\mathrm{SM}}$}

In this section we discuss the salient features of the NMSSM Higgs sector corresponding to the parameter space which leads to the second lightest $\mathrm{CP}$ even Higgs boson, $\mathrm{H}_{2}$, as SM-like. Here, the lightest $\mathrm{CP}$ even Higgs $\left(\mathrm{H}_{1}\right)$ lighter than $\mathrm{H}_{2}$ by definition. In addition the lightest

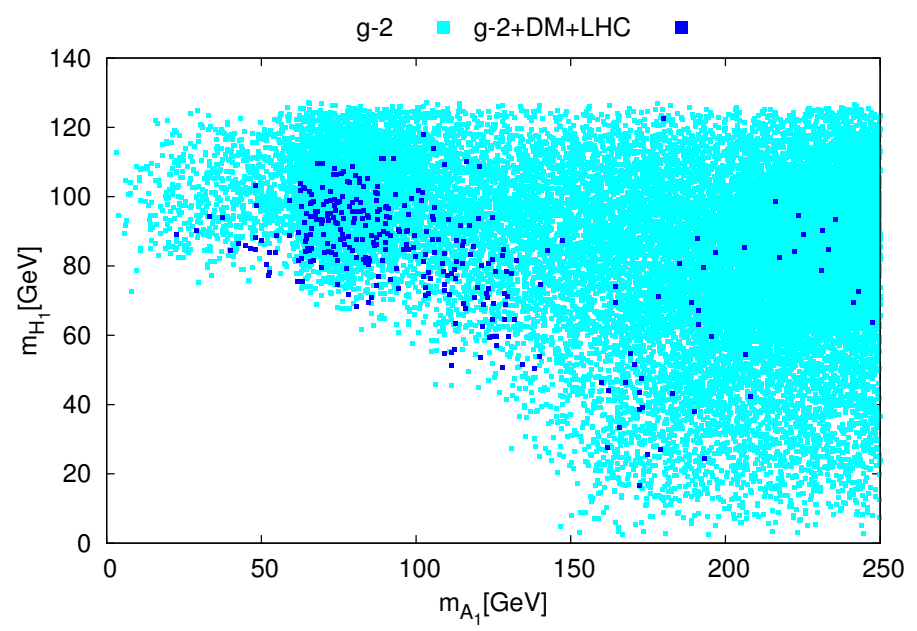

Figure 1: Range of $\mathrm{m}_{\mathrm{A}_{1}}$ and $\mathrm{m}_{\mathrm{H}_{1}}$ for case(A), allowed by all theoretical and experimental constraints as discussed in Sec.3 plus $g_{\mu}-2$ constraint(cyan) and then adding the LHC and DM constraints(blue).

$\mathrm{CP}$ odd Higgs boson $\left(\mathrm{A}_{1}\right)$ is also lighter than $\mathrm{H}_{2}$ in a certain region of parameter space. Both $H_{1}$ and $A_{1}$ are dominantly singlet like. In Fig. 1, we present the possible ranges of $\mathrm{m}_{\mathrm{H}_{1}}$ and $\mathrm{m}_{\mathrm{A}_{1}}$ when $H_{2}$ is SM-like [24]. In this figure the allowed points (cyan) are result from imposing 
only the $g_{\mu}$-2 limit (Eq 3.21 along with all other theoretical and experimental constraints as described in the previous section. Subsequently, adding constraints from the DM relic density (Eq. 3.18) and from the measurements of the Higgs couplings at the LHC (Table 1), the more restricted region(blue) is obtained. It is interesting to note that, even after including all such constraints, $H_{1}$ or $A_{1}$ may turn out to be very light, $\sim 20 \mathrm{GeV}$, although there are only few points 76,77. Moreover, this figure clearly indicates that for a good fraction of the parameter space, both $\mathrm{m}_{\mathrm{H}_{1}}$ and $\mathrm{m}_{\mathrm{A}_{1}}$ are found to be less than the half of the mass of $\mathrm{H}_{2}$, thus allowing the latter decay in the following ways,

$$
\begin{aligned}
\mathrm{H}_{2} & \rightarrow \mathrm{H}_{1} \mathrm{H}_{1}, \\
& \rightarrow \mathrm{A}_{1} \mathrm{~A}_{1} .
\end{aligned}
$$

It is to be noted that these lighter singlet states $\mathrm{A}_{1}$ and $\mathrm{H}_{1}$ are not yet ruled out by any collider experiments. These singlet like states escape detection in collider experiments because of their suppressed couplings to gauge bosons and fermions. It can be realized by looking at Figs 2 and
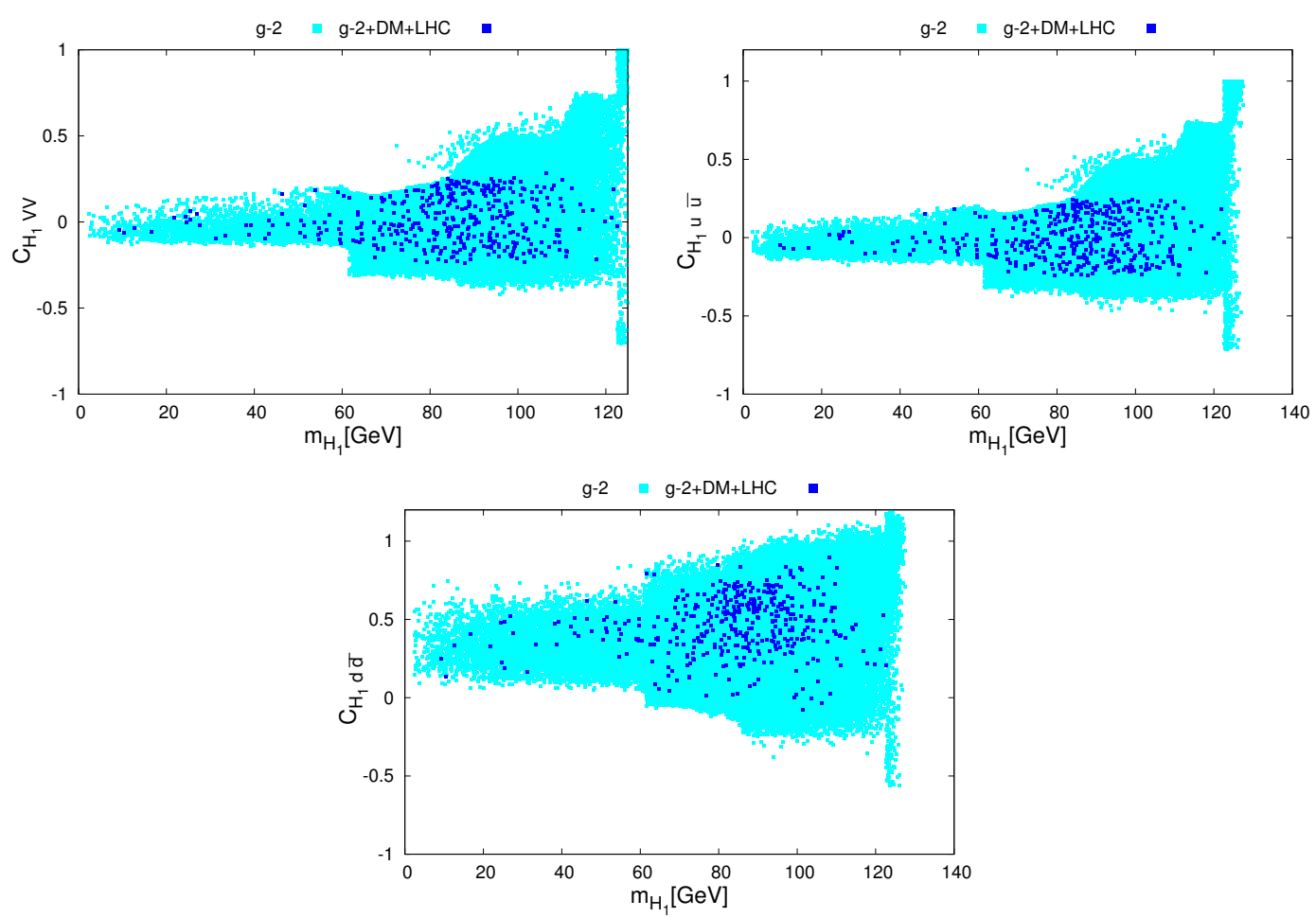

Figure 2: Reduced couplings of $\mathrm{H}_{1}$ with gauge bosons $(\mathrm{V}=\mathrm{W} / \mathrm{Z})$ and fermions (up and down types) for case(A). Values are allowed by mentioned constraints ( conventions are the same as in Fig. 1.)

3 which present for a wide range of $m_{H_{1}}$, the reduced couplings of $\mathrm{H}_{1}$ and $\mathrm{A}_{1}$, respectively, with gauge bosons, up and down-type quarks. At lower masses for $\mathrm{H}_{1}$, allowed values of these reduced couplings initially appear to be very small and then go up with increasing $\mathrm{m}_{\mathrm{H}_{1}}$. However, LHC constraints, in particular restrictions on reduced couplings of the SM-like Higgs boson as shown in Table 1 , favor smaller values, except for the case $\mathrm{C}_{\mathrm{H}_{1} \mathrm{~d} \overline{\mathrm{d}}}$ which receives some enhancement for higher values of $\tan \beta$ (see Eq. 2.7). In case of $A_{1}$, the pattern of variation 
of reduced the couplings with $\mathrm{m}_{\mathrm{A}_{1}}$ is a little different since $\mathrm{A}_{1}$ physical state contains a finite fraction of doublet component and the reduced couplings increase for higher values of $\tan \beta$. As observed in Fig. 3. the reduced coupling of $A_{1}$ to d-type quark is somewhat larger than its coupling to u-type quark. Consequently, this behaviour of the reduced couplings significantly affects the Higgs phenomenology in the colliders. These are discussed in the next section.
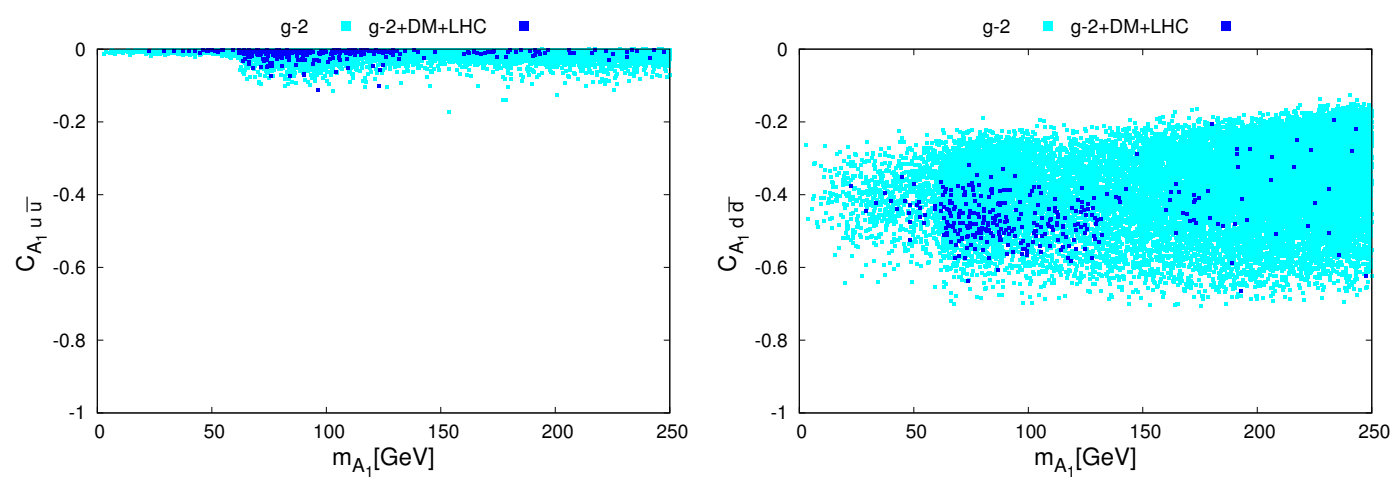

Figure 3: Reduced couplings of $\mathrm{A}_{1}$ with quarks (up and down types). Other conventions are same as in Fig. 2 .

\subsection{Case B: $\mathrm{H}_{1} \sim \mathrm{H}_{\mathrm{SM}}$}

Following a similar strategy as described above, we obtain a substantial region of parameter space with the lightest $\mathrm{CP}$ even state $\mathrm{H}_{1}$ as the SM-like Higgs boson. Obviously, in this

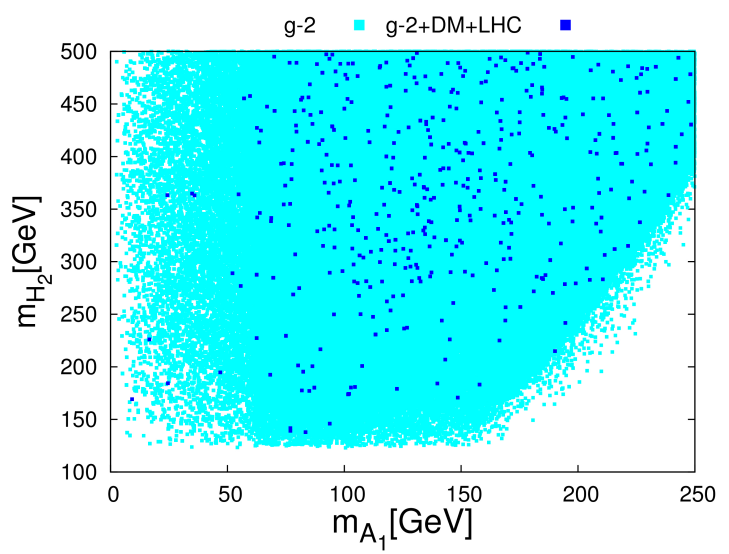

Figure 4: Allowed range of $m_{A_{1}}$ and $m_{H_{2}}$ for case (B). Constraints are the same as in Fig. 1 .

scenario, $\mathrm{m}_{\mathrm{H}_{2}} \gtrsim \mathrm{m}_{\mathrm{H}_{1}} \sim \mathrm{m}_{\mathrm{H}_{\mathrm{SM}}}$ and the mass of $\mathrm{A}_{1}$ can be lighter or heavier than $\mathrm{H}_{1}$. In fact, a significant region of parameter space exists even after imposing all constraints where it is observed that $\mathrm{A}_{1}$ is light, around $\sim 50-100 \mathrm{GeV}$ and it can turn out to be even lighter $\sim 10 \mathrm{GeV}$ for few points( see Fig. 4), where allowed ranges of $m_{A_{1}}$ and $m_{H_{2}}$ are presented for this scenario. This figure indicates that the available range of $m_{A_{1}}$ can be lighter than $m_{H_{1}} / 2$, whereas $m_{H_{2}}$ may extend to very large $(\sim 500 \mathrm{GeV})$ values. Hence the following 
decay channels,

$$
\begin{aligned}
& \mathrm{H}_{2} \rightarrow \mathrm{H}_{1} \mathrm{H}_{1}, \mathrm{~A}_{1} \mathrm{~A}_{1}, \\
& \mathrm{H}_{1} \rightarrow \mathrm{A}_{1} \mathrm{~A}_{1},
\end{aligned}
$$

open up with a reasonable branching ratio depending on the region of the parameter space. These decay channels may potentially provide additional indirect sources of productions of $\mathrm{H}_{1}$ as well as of $\mathrm{A}_{1}$ at the LHC mediated by triple Higgs bosons couplings.
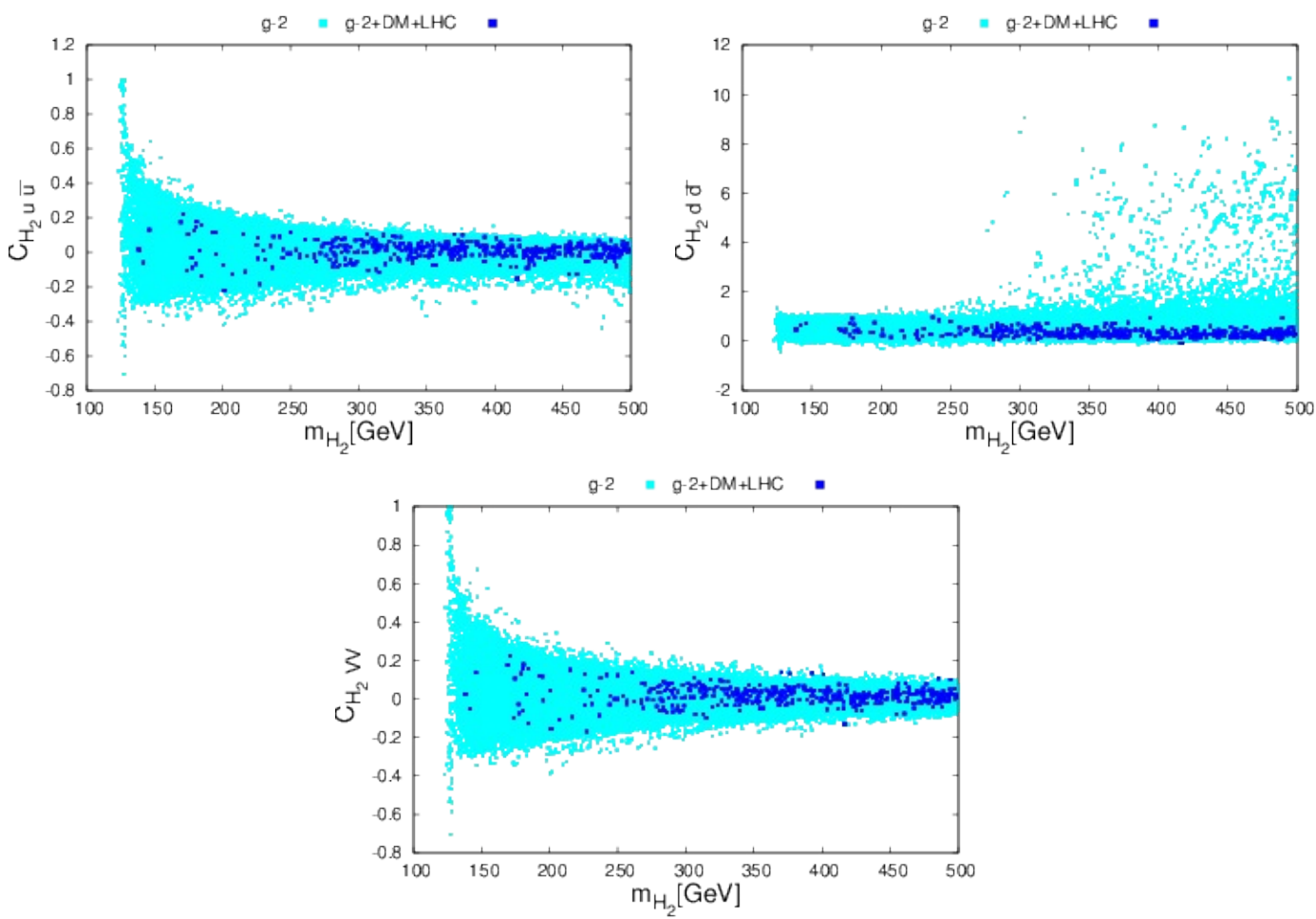

Figure 5: Reduced couplings of $\mathrm{H}_{2}$ with gauge bosons $(\mathrm{V}=\mathrm{W} / \mathrm{Z}$ ) and quarks( up and down types) for case(B). Constraints are the same as Fig. 1 .
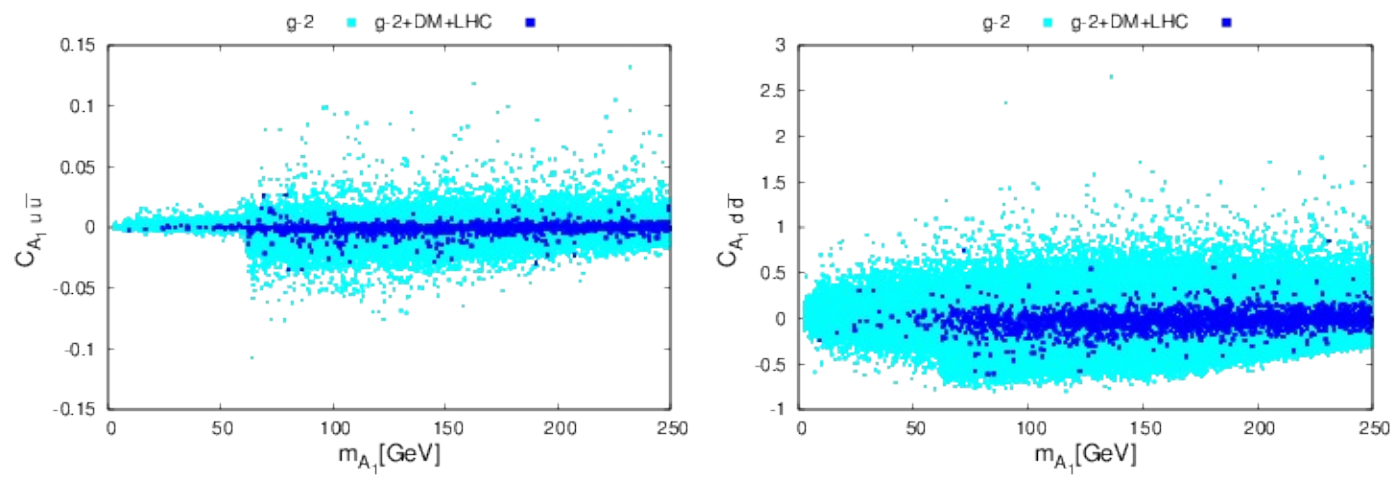

Figure 6: Same as in Fig. 3, but for case(B). 
As before, in order to understand the phenomenology in this scenario of non SM singletlike $\mathrm{H}_{2}$ and $\mathrm{A}_{1}$, we investigate the coupling strengths of these Higgs states. Since $\mathrm{H}_{1}$ is SM-like, and it predominantly couples to gauge bosons, obviously the sum rule (Eq. 2.10) predicts suppression of the corresponding reduced couplings of $\mathrm{H}_{2}$, as shown in Fig. 5 (bottom panel). Notice that the reduced couplings of $\mathrm{H}_{2}$ with d-type quark are reasonably large, $\sim 2$ 10 depending on the masses, but additional DM and LHC constraints restrict those to lower values. The variation of the reduced couplings of $A_{1}$ is also studied and is shown in Fig. 6. The reduced couplings of $\mathrm{A}_{1}$ to the $\mathrm{u}$-type quark is almost negligible whereas with the d-type quark it has finite values. Moreover, because of the absence of $\mathrm{A}_{1}$ coupling to gauge bosons, direct production of $\mathrm{A}_{1}$ in lepton colliders and via vector boson fusion in hadron colliders are suppressed. Hence, the low masses of $A_{1}$ are not excluded by LEP experiment. However, in hadron colliders $A_{1}$ can be produced via gluon gluon fusion or $\mathrm{b}-\overline{\mathrm{b}}$ annihilation and due to the suppressed couplings of $A_{1}$ with u-type quarks, the latter process is the comparatively dominant one.

Armed with this knowledge about the spectrum of allowed masses and the couplings of the light neutral non SM Higgs bosons for the two scenarios, (A) and (B), we now discuss their decay patterns and followed by their phenomenological implications at the LHC.

\subsection{Higgs decays: $\mathrm{H}_{1}, \mathrm{~A}_{1}, \mathrm{H}_{2}$}

As pointed out earlier, the NMSSM Higgs bosons offer a rich phenomenology at colliders owing to their very diverse decays in various channels including some non SM modes. Furthermore, the BR of each Higgs decay modes and hence, the sensitivity of the corresponding signal, are very much parameter space dependent because of the presence of complicated admixtures of physical Higgs states making them either doublet- or singlet-like. In this work, in order to predict NMSSM Higgs signal at the LHC in the context of the scenarios presented in case(A) and case(B), we revisit various Higgs decay channels by studying correlations among the BRs and the parameter space. We also focus on a few interesting Higgs decay modes which can lead to robust signals of the NMSSM Higgs bosons, in particular, for the lightest CP odd and the $\mathrm{CP}$ even Higgs bosons. In the present context, we discuss features of BRs of various decay channels only for two $\mathrm{CP}$ even $\left(\mathrm{H}_{1}, \mathrm{H}_{2}\right)$ and the lightest $\mathrm{CP}$ odd $\left(\mathrm{A}_{1}\right)$ Higgs bosons.

- $\mathrm{H}_{1}$ decays : As mentioned before, for certain regions of the parameter space in the scenario represented by case(A) with $\mathrm{H}_{2}$ as the SM-like Higgs boson, $\mathrm{H}_{1}$ and $\mathrm{A}_{1}$ can be very light and can have masses below $50 \mathrm{GeV}$, see Fig 1. The decay $\mathrm{H}_{1} \rightarrow \mathrm{A}_{1} \mathrm{~A}_{1}$ will be the dominant one whenever it is kinematically allowed. The corresponding decay width is primarily determined by triple Higgs $\mathrm{H}_{1}-\mathrm{A}_{1}-\mathrm{A}_{1}$ coupling mediated by singlet components and approximately proportional to $\kappa v_{s}$, thus making the decay width very large in comparison to other fermionic and bosonic channels. It is found that for certain set of parameters, the $\mathrm{BR}\left(\mathrm{H}_{1} \rightarrow \mathrm{A}_{1} \mathrm{~A}_{1}\right)$ goes to $90 \%$ or more. As expected, this triple Higgs boson coupling receives corrections 78,79 which are not taken into account in this present calculation. There exists a subset of parameter space where $\mathrm{H}_{1}$ decays dominantly to $b \bar{b}$ channel with $\mathrm{BR}$ close to $90 \%$ and rest to the $\tau \tau$ channel $(\sim 10 \%)$ as shown in Fig. 7(left) for various masses of $H_{1}$. Although the $\mathrm{H}_{1}$ state is dominated by the singlet component, presence of a finite fraction of doublet component $\left(\mathrm{S}_{\mathrm{i} 2}\right.$, composition of $\mathrm{H}_{\mathrm{d}}$ ) leads to a sizeable coupling of $H_{1}$ to d-type fermions (Eq2.7). As a consequence, the partial widths of $\mathrm{H}_{1} \rightarrow \mathrm{b} \bar{b}, \tau \tau$ modes turn out to be dominant leading to enhancements of respective BRs. 

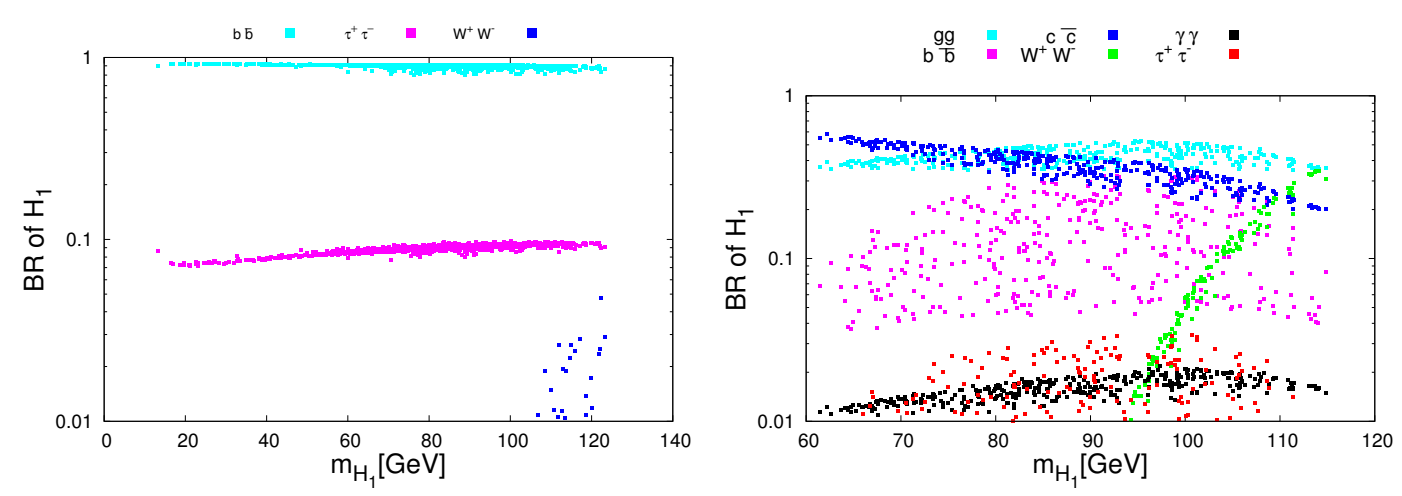

Figure 7: $\mathrm{BR}$ of $\mathrm{H}_{1}$ in different channels for case(A) and allowed by all constraints including $g_{\mu}-2$, LHC and DM.

On the other hand, for certain region of the parameter space (where $m_{H_{1}} \gtrsim 60 \mathrm{GeV}$ ), the coupling $C_{H_{1} u \bar{u}}$ becomes finite (see Fig.2) resulting in an enhancement of the decay width of $H_{1} \rightarrow g g$ (via top quark), $c \bar{c}$ channels leading a little suppression of $b \bar{b}$ decay BR as demonstrated in Fig. 7 (right), provided Higgs to Higgs decays are kinematically forbidden. Notice that for the mass range around $\mathrm{m}_{\mathrm{H}_{1}} \sim 60 \mathrm{GeV}$ or above, the gg, $c \bar{c}$ channels are quite large [80]. A finite fraction of about $4-6 \%$ of $H_{1}$ decays to $W W$ for higher $\mathrm{m}_{\mathrm{H}_{1}}$ and also $\sim 2-3 \%$ $\mathrm{BR}$ is found for the $\gamma \gamma$ channel [81]. Note that the pattern of BR as shown in Fig. 7)(left and right) correspond to two different regions of the parameters space. For case(B), $\mathrm{H}_{1}$ is SM-like and decays via various SM channels with almost the same rate as in the SM. In addition, it can also decay to some non SM modes, such as $\mathrm{H}_{1} \rightarrow \mathrm{A}_{1} \mathrm{~A}_{1}, \tilde{\chi}_{1}^{0} \tilde{\chi}_{1}^{0}$ whenever kinematically allowed. Note that, however, as mentioned earlier, the total BR to these non SM channels of SM-like $\mathrm{H}_{1}$ is restricted by an upper bound, as given by Eq. 3.23. Perhaps, looking for these non SM decay channels of the SM-like Higgs can be a potential avenue to confirm the existence of this type of SUSY scenario.

- $\mathrm{A}_{1}$ decays : For the scenario presented by case $(\mathrm{A})$, the lightest $\mathrm{CP}$ odd Higgs boson $\mathrm{A}_{1}$ primarily decays to fermions due to the absence of tree level couplings with gauge bosons. As

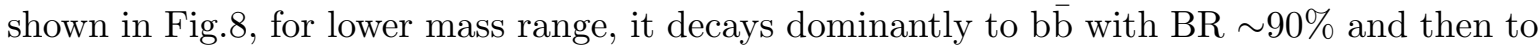
sub-dominant $\tau \tau$ channels. However, if decay modes such as, $\mathrm{A}_{1} \rightarrow \tilde{\chi}_{1}^{0} \tilde{\chi}_{1}^{0}, \tilde{\chi}_{1}^{0} \tilde{\chi}_{2}^{0}$ are accessible for a heavier $A_{1}(\gtrsim 150 \mathrm{GeV})$, then those turn out to be the dominant ones due to the enhancement of $\mathrm{A}_{1}$ couplings with singlino-Higgsino components in $\tilde{\chi}_{1}^{0}, \tilde{\chi}_{2}^{0}$ states leading to larger width. Here, $\tilde{\chi}_{1}^{0}$ and $\tilde{\chi}_{2}^{0}$ are the lightest and the second lightest neutralinos respectively. In this scenario, the $\mathrm{BR}$ of $\mathrm{A}_{1}$ in the di-muon final state is very tiny $\left(\sim 10^{-4}\right)$ as shown in the same figure along with other sub dominant decay modes such as, $\mathrm{A}_{1} \rightarrow \gamma \gamma$, gg, $\mathrm{Z} \gamma, \mathrm{ZH}_{1}$. For the scenario where $\mathrm{H}_{1}$ is SM-like i.e.in case(B), the BRs of $A_{1}$ to various final states are depicted in Fig. 9. Interestingly, note that $\mathrm{BR}\left(\mathrm{A}_{1} \rightarrow \gamma \gamma\right)$ can indeed be very large $(\sim 100 \%)$ and even greater than the BR for $b \bar{b}$ channel, in certain regions of the parameter space and for a wide range of $m_{A_{1}}$ (left). This interesting decay pattern of $\mathrm{A}_{1}$ in the di-photon channel can be understood by more careful investigation of the structures of $A_{1}$ couplings to various particles. 

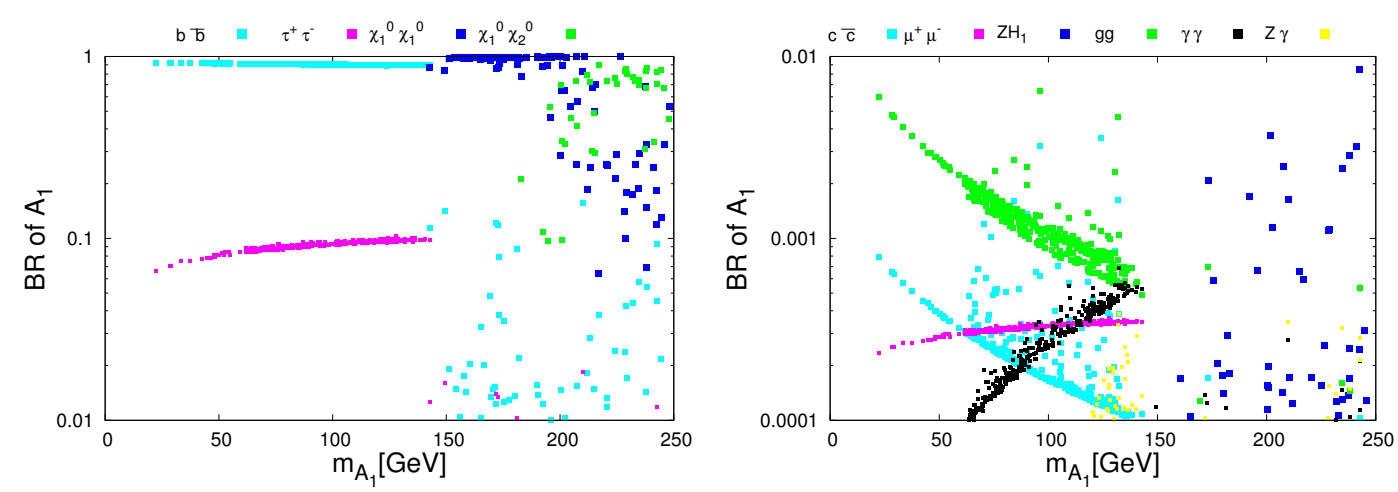

Figure 8: BR of $A_{1}$ for case(A), same as in Fig. 7.
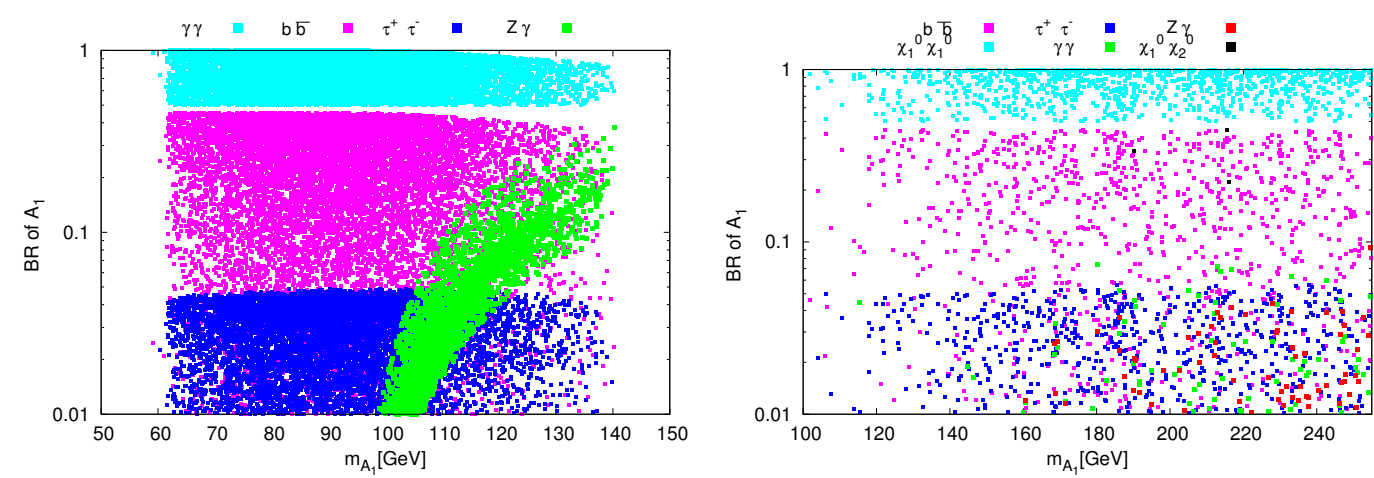

Figure 9: Same as in Fig. 8 but for case(B).

Here, note that $A_{1}$ is dominantly singlet like resulting in all of its available fermionic decay channels suppressed. Primarily the off-diagonal elements in the Higgs mass matrix determine the composition of Higgs boson states. For example, in case of $A_{1}$ when the off-diagonal term of pseudoscaler Higgs mass matrix vanishes for a certain combination of the parameters, then $A_{1}$ becomes completely singlet like. From the $2 \times 2$ Higgs $\mathrm{CP}$ odd mass matrix, one can conclude that the vanishing of off-diagonal term leads $A_{1}$ purely singlet dominated which can be translated to the relation such as $A_{\lambda} \sim \frac{2 \kappa \mu_{e f f}}{\lambda}$ and it occurs for a wide region of parameters. Moreover, $A_{1}$ does not have any tree level coupling with the vector bosons. In this kind of a scenario, $\mathrm{A}_{1}$ can still couple to photons at one loop with charginos in the loop. The Higgsino like charginos favourably couple to a singlet like $\mathrm{A}_{1}$ state and enhance the partial width of $\mathrm{A}_{1} \rightarrow \gamma \gamma$ mode. Therefore, for the regions of the parameter space where both the charginos are Higgsino like, $\mathrm{BR}\left(\mathrm{A}_{1} \rightarrow \gamma \gamma\right)$ turns out to be very large for a wide range of $\mathrm{m}_{\mathrm{A}_{1}}$ as clearly seen in Fig. 9 23, 82. Evidently, this di-photon final state appears to be one of the striking features of this model and can be exploited not only to discover $\mathrm{A}_{1}$ at the LHC, but also to discriminate the MSSM from the NMSSM. It will be discussed later in more detail. In addition, $\mathrm{A}_{1} \rightarrow \tau \tau$ mode is also present with a reasonable $\mathrm{BR}(\sim 10 \%)$ which can also provide a clean signal of $\mathrm{A}_{1}$. The other sub dominant decay modes of $A_{1}$ are presented in Fig. 9 (right) where the dominant one is the invisible decay channel, $A_{1} \rightarrow \tilde{\chi}_{1}^{0} \tilde{\chi}_{1}^{0}$. The BR of $\mathrm{A}_{1}$ in the muonic channel, $\mathrm{A}_{1} \rightarrow \mu \mu$ is of the same order $\left(\sim 10^{-4}\right)$ as before along with other subdominant channels like $\mathrm{A}_{1} \rightarrow \mathrm{gg}, \mathrm{Z} \gamma$. Note that, both the plots in Figs. 8 and 9 presenting 
the BRs of $A_{1}$ and $H_{1}$ respectively correspond to two different regions of the parameter space for each cases.

- $\mathrm{H}_{2}$ decays: In the scenario described in case(B), $\mathrm{H}_{2}$ can decay to a pair of SM-like $\mathrm{H}_{1}$ as well as to a singlet like $\mathrm{A}_{1}$ pair i.e. $\mathrm{H}_{2} \rightarrow \mathrm{H}_{1} \mathrm{H}_{1}, \mathrm{~A}_{1} \mathrm{~A}_{1}$. If kinematically allowed, these are expected to be dominant $(\sim 80-100 \%)$ is clearly shown in Fig. 10 (left). Since, $\mathrm{H}_{1}$ is SM-like, the decay channel, $\mathrm{H}_{2} \rightarrow \mathrm{H}_{1} \mathrm{H}_{1}$ is likely to contribute to the rate of $\mathrm{SM}$ di-Higgs production at the LHC. Similarly, in Fig 10 (right) we present BRs of various decay channels of $\mathrm{H}_{2}$ for case $(\mathrm{A})$.

In this case, notice that for a good fraction of parameter space the mass of $\mathrm{H}_{2} \sim \mathrm{H}_{\mathrm{SM}}$ is heavier than that of $H_{1}$ and $A_{1}$ (see Fig 1 ), and $\mathrm{BR}\left(\mathrm{H}_{2} \rightarrow \mathrm{H}_{1} \mathrm{H}_{1}\right)$ is found to vary between a few percent to $\sim 40 \%$ where as $\operatorname{BR}\left(H_{2} \rightarrow \mathrm{A}_{1} \mathrm{~A}_{1}\right)$ is enhanced to $30 \%$. Note that, as before, these are the non SM decay modes of the SM-like Higgs $\mathrm{H}_{2}$ and also allowed by the constraint in Eq. 3.23. However, the decay channel $\mathrm{H}_{2} \rightarrow \mathrm{H}_{1} \mathrm{H}_{1}$ is expected to contribute to the visible signal of the SM-like Higgs boson production. Hence, it is worth examining the contribution of this decay channel in the present Higgs data collected at LHC Run I experiments, which eventually may constrain the model [83]. Notice that in this scenario, case(A), $\mathrm{H}_{2}$ has a reasonable BR ( $\sim 10-90 \%)$ to a pair of lightest neutralinos contributing to its invisible decay width 84. In addition, $\mathrm{H}_{2}$ has many other sub dominant decay modes which can provide interesting signals, in particular for the scenario (B). For example, decays to SUSY particles such as a pair of neutralinos, $\mathrm{H}_{2} \rightarrow \tilde{\chi}_{2}^{0} \tilde{\chi}_{2}^{0}, \tilde{\chi}_{2}^{0} \tilde{\chi}_{3}^{0}$, may provide clean signals because of leptonic decays of the neutralinos. We found that in this regions of the parameter space, $\mathrm{H}_{2} \rightarrow \mathrm{ZA}_{1}$ is very tiny.
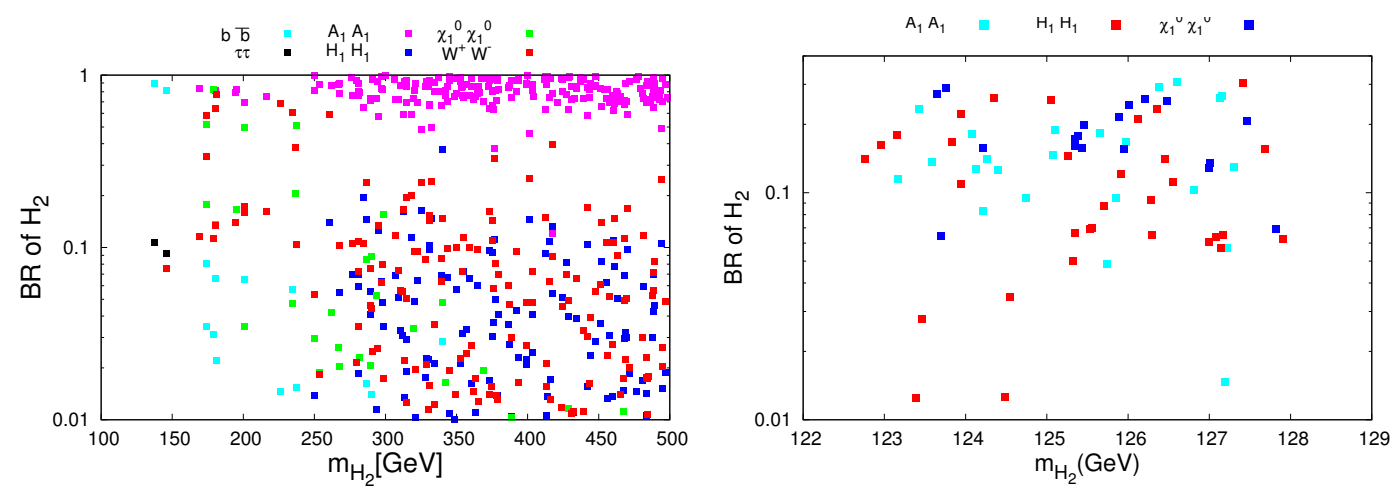

Figure 10: $\mathrm{BR}$ of $\mathrm{H}_{2}$ for case(B)(left) and case(A)(right) subject to all constraints as in Fig 7.

\section{Higgs production at the LHC}

In general, the NMSSM Higgs bosons can be produced via conventional mechanisms, i.e by the dominant gluon-gluon fusion ( $\mathrm{ggF}$ ), whereas the next sub dominant mode is the associated productions with a pair of $\mathrm{b}$ quarks. However for a very large value of $\tan \beta$, the associated production mode appears to be the dominant one. We reiterate here that in the NMSSM, the Higgs couplings to fermions and the gauge bosons are essentially the SM couplings scaled by the respective reduced coupling factors which can vary widely from small $(\ll 1)$ to moderately large values $(\sim 0.5)$. It implies that the Higgs boson production cross-section in the NMSSM can be obtained from the corresponding SM cross-section by appropriately rescaling them by 
the respective factors. Hence the following naive strategy can be adopted to estimate the NMSSM Higgs boson production cross section as 24, 85,

$$
\sigma_{\mathrm{ggF}}(\phi)=\sigma_{\mathrm{ggF}}^{\mathrm{SM}}(\phi) \times \mathrm{C}_{\mathrm{gg}}^{2},
$$

where $\phi$ stands for any of the CP even or odd Higgs boson states. Here $\sigma_{\operatorname{ggF}}(\phi)$ is the NMSSM Higgs boson production cross section via gluon-gluon fusion and $\sigma_{\mathrm{ggF}}^{\mathrm{SM}}(\phi)$ is the corresponding SM cross section for the same Higgs boson mass. $\mathrm{C}_{\mathrm{gg}}$ is the effective reduced coupling gg $\phi$ via loops comprising heavier quarks and squarks. The SM Higgs boson production cross-section through ggF is known at the level of next-to-next-to leading order(NNLO) QCD [86 92]. We compute these SM Higgs boson production cross section via ggF using SusHi-v1.5.0 93 which takes into account the QCD contribution up to NNLO mediated by only the SM particles.

However, it is to be noted that in SUSY model, the ggF channel may receive a sizeable contribution from loops involving the corresponding superpartners, in particular, the third generation squarks, the stops and the sbottoms 94 . Recently, in the context of the NMSSM, the neutral Higgs boson production cross-section via ggF is computed including NLO contributions of the squarks and the gluino in addition to the electroweak corrections involving light quarks and it is implemented in SusHi [25]. These calculations are based on the expansion of terms of inverse of heavy SUSY particle masses 95 97. Obviously, numerical estimation using this calculation is expected to be more reliable than the approximate method described in Eq. 4.27. Hence, in this work we use this calculation implemented in SusHi-v1.5.0 93 to compute the neutral Higgs boson production cross sections in the NMSSM. Thus, the production rate of Higgs decaying to any of the pair of state $\mathrm{X}$ can be estimated as,

$$
\mathrm{R}_{\mathrm{XX}}=\sigma_{\mathrm{ggF}}(\phi) \times \mathrm{BR}(\phi \rightarrow \mathrm{XX}),
$$

where $\phi \rightarrow \mathrm{XX}$ collectively represents the Higgs decay channels with $\mathrm{XX}=\mathrm{b} \overline{\mathrm{b}}, \tau \tau, \gamma \gamma$, gg, $\mathrm{c} \overline{\mathrm{c}}, \mu \mu$, WW, ZZ etc. For a given decay channel and a set of parameter values, $\mathrm{BR}(\phi \rightarrow \mathrm{XX})$ is obtained from the NMSSMTools. $\sigma_{\mathrm{ggF}}(\phi)$ is computed directly using the NLO calculation 25] in the NMSSM implemented in SusHi. On the other hand, for the sake of comparison, we also estimate the cross section for the same Higgs boson production with the same mass following the approximate method as described in Eq. 4.27. It is found that following this prescription the calculated CP even Higgs boson production cross sections are 30-40\% higher than the value obtained using a more exact calculation of SusHi [25]. This can be attributed to the fact that in the NMSSM, the production cross sections are estimated at NLO including electroweak corrections, where as in Eq. 4.27 NNLO QCD result without any SUSY loops are used along with $\mathrm{C}_{\mathrm{gg}}$ estimated at NLO.

The cross-section for the next-dominant production mode, can be obtained by exclusive Higgs boson production in association with a pair of b quarks [98],

$$
\mathrm{gg} \rightarrow \mathrm{b} \overline{\mathrm{b}} \phi
$$

However, this cross section can be obtained also by computing $b \bar{b} \rightarrow \phi$ which is a good approximation within the higher order correction [99, 100]. Note that the SusHi calculates this $\mathrm{b} \overline{\mathrm{b}} \rightarrow \phi$ at NNLO QCD. The rate $\mathrm{R}_{\mathrm{XX}}$ corresponding to this production channel is obtained by multiplying the effective reduced $\mathrm{b} \overline{\mathrm{b}} \phi$ coupling factor with the SM production cross section obtained from SuSHi 93 and the respective $\operatorname{BR}(\phi \rightarrow X X)$. In our calculation we use the CT10 101] for parton distributions function and both the QCD scales are set to $\mathrm{M}_{\phi}$ for $\mathrm{ggF}$, 
whereas the factorization scale is fixed to $0.25 \mathrm{M}_{\phi}$ only for $\mathrm{b} \overline{\mathrm{b}} \rightarrow \phi$ production. Needless to say that the rate of the Higgs production in any of the decay channels is very much sensitive to the model parameters owing to the presence of mixing angles in the Higgs couplings, see Eq. 2.7 and 2.8 .

Indirectly, $\mathrm{H}_{1}$ and $\mathrm{A}_{1}$ can also be indirectly produced in pairs through $\mathrm{H}_{2}$ production via ggF and its subsequent decays provided $\mathrm{m}_{\mathrm{H}_{1}}, \mathrm{~m}_{\mathrm{A}_{1}}<\mathrm{m}_{\mathrm{H}_{2}} / 2$. The corresponding production rate can be obtained as,

$$
\sigma_{\mathrm{ggF}}^{\phi \phi}\left(\mathrm{H}_{2}\right)=\sigma_{\mathrm{ggF}}\left(\mathrm{H}_{2}\right) \times \mathrm{BR}\left(\mathrm{H}_{2} \rightarrow \phi \phi\right)
$$

where $\phi=\mathrm{H}_{1}, \mathrm{~A}_{1}$. As before, the $\sigma_{\mathrm{ggF}}\left(\mathrm{H}_{2}\right)$ is computed from NLO calculation in the NMSSM framework using SusHi 25].

Now we present the light neutral non-SM Higgs bosons production rates $\mathrm{R}_{\mathrm{XX}}$ (Eq. 4.28) via both the production modes, ggF and $\mathrm{b} \overline{\mathrm{b}} \rightarrow \phi$ for various decay modes corresponding to the regions of parameter space presented by case $(\mathrm{A})$ and case $(\mathrm{B})$. As pointed out earlier, the opening up of many decay modes of the non-SM-like Higgs bosons offers a variety of signals with a widely ranging rates. In order to demonstrate, we compute the minimum and the maximum values of those rates, $\mathrm{R}_{\mathrm{Xx}}$ for those region of the parameter space for a given mass of the Higgs boson and the decay channel. Naively, such regions of the parameter space which leads to the minimum and maximum cross sections for a given Higgs mass and decay channel are identified by the corresponding values of the product, $\mathrm{C}_{\mathrm{gg}}^{2} \times \mathrm{BR}(\phi \rightarrow \mathrm{XX})$. We assume that the parameter region which gives the maximum(minimum) value of this product yield a maximum(minimum) value of the cross section for the given Higgs boson mass and the decay channel. In Tables 2 - 4, we present minimum and maximum rates for non-SM-like Higgs bosons in various decay channels for the scenarios case(A) and case(B). For example, in Table 2, the results are shown for the non-SM-like $\mathrm{H}_{1}$ for case(A). Rates are presented for the Higgs production via both $\operatorname{ggF}\left(R_{X X}(g g)\right)$ and associated production, $b \bar{b} \rightarrow \phi\left(R_{X X}(b \bar{b})\right)$. In this table, the first row shows the selected values of $m_{H_{1}}$ extending up to $120 \mathrm{GeV}$, while the first column indicates the rates $\left(\mathrm{R}_{\mathrm{XX}}\right)$ for the decay channel $\mathrm{XX}$ of which minimum and maximum values are presented. For instance, with a choice of lower value of $m_{H_{1}}=13 \mathrm{GeV}$, the maximum rate of $\mathrm{H}_{1}$ production in $b \bar{b}$ channel via $\mathrm{ggF}$ is about $145 \times 10^{3} \mathrm{fb}$, where as the same for $\gamma \gamma$ and $\tau \tau$ channels are about $1.4 \mathrm{fb}$ and $14 \times 10^{3} \mathrm{fb}$, respectively. As seen in this Table that the rates $\left(\mathrm{R}_{\mathrm{XX}}(\mathrm{b} \overline{\mathrm{b}})\right)$ via associated production Higgs are also not negligible. Note that we do not present the minimum values of the cross sections if it is found to be less than $10^{-4} \mathrm{fb}$. Also, we only present single numbers for those cases where we have very few allowed points in the parameter space and no presentable variation in rates is observed for them. It is well known that the signals in the $\gamma \gamma, \tau \tau$ channels are comparatively clean due to the efficient tagging of photon and improved detection of $\tau$ leptons either in jets or leptonic final states. For higher values of $\mathrm{m}_{\mathrm{H}_{1}}$, say for $110 \mathrm{GeV}$, the dominant cross section is found to be again for the $b \bar{b}$ final state values ranging over from a few fb to a few thousands fb, whereas in the case of the $\tau \tau$ final state, the maximum value lies within a few $100 \mathrm{fb}$. Interestingly, note that the rate of the $\mathrm{H}_{1}$ production decaying in the $\mathrm{H}_{1} \rightarrow$ gg channel is reasonably large for all masses, unlike the situation in the SM as well as in the MSSM, where it is very much suppressed. Hence this channel can have the potential candidate to distinguish the NMSSM from other SUSY models, although elimination of QCD background is a non-trivial task. Remarkably, we observe that at higher values of $\mathrm{m}_{\mathrm{H}_{1}}$ for which $\mathrm{m}_{\mathrm{A}_{1}} \lesssim \mathrm{m}_{\mathrm{H}_{1}} / 2$, Higgs to Higgs decays, such as $\mathrm{H}_{1} \rightarrow \mathrm{A}_{1} \mathrm{~A}_{1}$ open up with a maximum rate found to be $\mathcal{O}(1000 \mathrm{fb})$ which is not shown 


\begin{tabular}{|c|ccccccc|}
\hline Rates & \multicolumn{7}{c}{$m_{H_{1}}(\mathrm{GeV})$} \\
& 13 & 28 & 48 & 68 & 89 & 110 & 120 \\
\hline$R_{b \bar{b}}(g g)$ & 144927 & $2383-37088$ & $1164-9835$ & $126-4735$ & $4.5-4345$ & $17.4-2337$ & $45-1126$ \\
$R_{b \bar{b}}(b \bar{b})$ & 1773 & $189-2952$ & $170-2133$ & 1868 & 868 & 421 & $6-249$ \\
\hline$R_{c \bar{c}}(g g)$ & 423 & $10-46$ & $1.3-60$ & $0.03-3737$ & $9 \mathrm{E}-4-1726$ & $4.5 \mathrm{E}-3-808$ & $0.07-11$ \\
$R_{c \bar{c}}(b \bar{b})$ & 5.18 & $0.8-3.15$ & $0.4-4.4$ & 4.3 & 3.2 & $3 \mathrm{E}-4-1.5$ & $3 \mathrm{E}-2-1.1$ \\
\hline$R_{\tau \tau}(g g)$ & 13838 & $213-2961$ & $105-858$ & $5 \mathrm{E}-3-455$ & $1.3 \mathrm{E}-2-448$ & $6.4 \mathrm{E}-2-250$ & $5-120$ \\
$R_{\tau \tau}(b \bar{b})$ & 169 & $17-236$ & $16-186$ & 180 & 87 & 44 & $0.61-27$ \\
\hline$R_{\mu \mu}(g g)$ & 55 & $0.76-11$ & $0.37-3$ & 1.6 & 1.6 & $2.2 \mathrm{E}-4-0.88$ & $1.7 \mathrm{E}-2-0.42$ \\
$R_{\mu \mu}(b \bar{b})$ & 0.7 & $0.06-0.85$ & $5.6 \mathrm{E}-2-0.66$ & 0.64 & 0.3 & 0.15 & $2.2 \mathrm{E}-3-9.6$ \\
\hline$R_{\gamma \gamma}(g g)$ & 1.4 & $0.01-0.47$ & $2.6 \mathrm{E}-3-1$ & $2.6 \mathrm{E}-4-93$ & 79 & $2 \mathrm{E}-4-58$ & $2.5 \mathrm{E}-4-0.88$ \\
$R_{\gamma \gamma}(b \bar{b})$ & $1.7 \mathrm{E}-2$ & $1 \mathrm{E}-3-3.2 \mathrm{E}-2$ & $7 \mathrm{E}-4-0.09$ & 0.17 & 0.22 & 0.18 & $1.2 \mathrm{E}-4-0.1$ \\
\hline$R_{g g}(g g)$ & 1028 & $12-161$ & $3-38$ & $0.22-2881$ & $5.6 \mathrm{E}-3-2077$ & $0.02-1351$ & $0.11-15$ \\
$R_{g g}(b \bar{b})$ & 12.6 & $0.93-12.8$ & $0.8-6$ & 4.8 & $1 \mathrm{E}-4-4.5$ & $4.3 \mathrm{E}-4-3$ & $4.3 \mathrm{E}-2-1.6$ \\
\hline
\end{tabular}

Table 2: Production rates(cross section $\times$ branching ratio) ( in $\mathrm{fb}$ ) of $\mathrm{H}_{1}$ at $13 \mathrm{TeV} \mathrm{LHC}$ energy for case(A).

here. In this case, of course, the possibility of observing the signal of $\mathrm{H}_{1}$ depends on $\mathrm{A}_{1}$ decay pattern and the measurement can provide the information about triple Higgs bosons coupling. It is also observed that the rate in the WW channel at the higher masses range $(\sim 120 \mathrm{GeV})$ is non negligible, and can vary between the range of $\mathcal{O}(1-100 \mathrm{fb})$. In addition, possibility of the $\mathrm{H}_{1} \rightarrow \tilde{\chi}_{1}^{\mathrm{o}} \tilde{\chi}_{1}^{0}$ (invisible decay) exists for higher $\mathrm{m}_{\mathrm{H}_{1}}(\gtrsim 100 \mathrm{GeV})$ with a very tiny rate in a certain region of parameter space.

Similarly in Table 3, we present the minimum and the maximum rates of $A_{1}$ in any given decay channel through both the production modes for a range of masses $22-249 \mathrm{GeV}$ for the scenario represented by the case(A). The dominant rates of $\mathrm{A}_{1}$ also appear to be in the $b \bar{b}$ decay channel. On the other hand, $\tau \tau, \gamma \gamma$ channels predict a range of sizeable rates, in particular for $\mathrm{m}_{\mathrm{A}_{1}} \sim 100 \mathrm{GeV}$ or less. For higher masses of $\mathrm{A}_{1}$, the rates for $\tau \tau, b \bar{b}$ channels are reasonable. Decays of $\mathrm{A}_{1}$ in various SUSY particles, in particular, for higher $A_{1}$ masses also appear with a few fb cross section which are not presented here. In Table 4 , as before, we present the rates of the $A_{1}$ production for a mass range from $17-249 \mathrm{GeV}$ in multiple channels for case(B) where $\mathrm{H}_{1}$ is the SM-like Higgs. Note that as before the experimentally clean $\gamma \gamma$ and $\tau \tau$ channels provide a sizeable rates for lower values of $\mathrm{m}_{\mathrm{A}_{1}}$, whereas for higher values of $\mathrm{m}_{\mathrm{A}_{1}}$, depending on the regions of the parameter space, the rates for $\tau \tau, b \bar{b}$ channels appear to be feasible for exploring $A_{1}$. The $c \bar{c}, g g$ channels are not very promising due to tiny rates and presence of large QCD background, except for the lower masses where the rates are sizeable. As observed before for the $\mathrm{H}_{1}$ case, the $b \bar{b}$ channel has the most dominant rates 


\begin{tabular}{|c|cccccccc|}
\hline Rates & \multicolumn{7}{c}{$m_{A_{1}}(\mathrm{GeV})$} & \\
& 22 & 30 & 60 & 100 & 140 & 180 & 218 & 249 \\
\hline$R_{b \bar{b}}(g g)$ & 72336 & 36759 & $818-1685$ & $143-226$ & $1.53-29$ & $0.08-0.3$ & $0.018-0.09$ & $9 \mathrm{E}-3-0.6$ \\
$R_{b \bar{b}}(b \bar{b})$ & 1392 & 2274 & $385-869$ & $115-262$ & $4-65$ & $0.3-1.1$ & $0.06-0.18$ & $0.04-0.06$ \\
\hline$R_{c \bar{c}}(g g)$ & 62 & 26 & $0.3-0.6$ & $1.7 \mathrm{E}-2-0.04$ & $1.6 \mathrm{E}-4-3.5 \mathrm{E}-3$ & $3.5 \mathrm{E}-5$ & - \\
$R_{c \bar{c}}(b \bar{b})$ & 1.19 & 1.57 & $0.13-0.3$ & $0.02-0.05$ & $4 \mathrm{E}-3-7 \mathrm{E}-3$ & - & - \\
\hline$R_{\tau \tau}(g g)$ & 5939 & 2813 & $76-156$ & $14-23$ & $0.17-3.2$ & $9 \mathrm{E}-3-3.4 \mathrm{E}-2$ & $2.2 \mathrm{E}-3-1.1 \mathrm{E}-2$ & $1 \mathrm{E}-3-7 \mathrm{E}-2$ \\
$R_{\tau \tau}(b \bar{b})$ & 99 & 174 & $36-82$ & $12-27$ & $0.5-7$ & $0.034-0.12$ & $0.007-0.02$ & $5 \mathrm{E}-3-7 \mathrm{E}-3$ \\
\hline$R_{\mu \mu}(g g)$ & 18.4 & 10 & $0.3-0.55$ & $0.05-0.08$ & $6 \mathrm{E}-4-1.2 \mathrm{E}-2$ & $1.1 \mathrm{E}-4$ & - \\
$R_{\mu \mu}(b \bar{b})$ & 0.35 & 0.62 & $0.12-0.3$ & $0.04-0.1$ & $0.002-0.02$ & $1 \mathrm{E}-4-4 \mathrm{E}-4$ & - \\
\hline$R_{\gamma \gamma}(g g)$ & 0.9 & 0.74 & $0.08-0.15$ & $0.04-0.07$ & $1 \mathrm{E}-3-1.7 \mathrm{E}-2$ & $1 \mathrm{E}-4-4 \mathrm{E}-4$ & $1.4 \mathrm{E}-4$ \\
$R_{\gamma \gamma}(b \bar{b})$ & 0.02 & 0.05 & $0.04-0.1$ & $0.03-0.09$ & $0.003-0.04$ & $3 \mathrm{E}-4-14 \mathrm{E}-4$ & $2 \mathrm{E}-4-3 \mathrm{E}-4$ \\
\hline$R_{g g}(g g)$ & 465 & 186.2 & $1.8-4.1$ & $0.14-0.3$ & $1 \mathrm{E}-3-2.2 \mathrm{E}-2$ & $1.1 \mathrm{E}-4$ & $(\mathrm{E}-4-2 \mathrm{E}-4$ \\
$R_{g g}(b \bar{b})$ & 9 & 11.5 & $0.9-1.8$ & $0.14-0.31$ & $0.003-0.05$ & $(1-4) \mathrm{E}-4$ & - \\
\hline
\end{tabular}

Table 3: Production rates(cross section $\times$ branching ratio)(fb) of $A_{1}$ at $13 \mathrm{TeV}$ LHC energy for $\operatorname{case}(\mathrm{A})$.

for all masses of $A_{1}$. The production rate of heavier non-SM-like Higgs boson $\mathrm{H}_{2}$ in various final states are presented in Table 5 for the case(B). For the lower mass range, just above the SM-like Higgs boson mass, the dominant rate is due to the $b \bar{b}$ mode as expected followed by the same in $\tau \tau$ channel which are not shown. For higher mass range around $\sim 200 \mathrm{GeV}$, the rates for the channels such as, $\mathrm{WW}, \mathrm{A}_{1} \mathrm{~A}_{1}$ and $\mathrm{H}_{1} \mathrm{H}_{1}$ are quite large. It is also observed the rate in the invisible decay mode $\left(H_{2} \rightarrow \tilde{\chi}_{1}^{0} \tilde{\chi}_{1}^{0}\right)$ is not negligible in a certain region of parameter space. Furthermore, for very high values $\left(m_{H_{2}} \geq 200 \mathrm{GeV}\right)$, Higgs to Higgs decay modes appear to be the dominant ones and provide a moderate rates ranging between a few $\mathrm{fb}$ to a few tens of a fb. 


\begin{tabular}{|c|cccccccc|}
\hline Rates & \multicolumn{7}{c}{$m_{A_{1}}(\mathrm{GeV})$} \\
& 17 & 30 & 59 & 100 & 138 & 181 & 220 & 249 \\
\hline$R_{b \bar{b}}(g g)$ & 36253 & 10164 & $.01-686$ & $6.3 \mathrm{E}-4-99$ & $4.5 \mathrm{E}-4-29$ & 7 & 0.55 & 4.8 \\
$R_{b \bar{b}}(b \bar{b})$ & 202 & 1058 & $0.004-263$ & $0.001-123$ & $0.001-33$ & 28 & 1.27 & 1.5 \\
\hline$R_{c \bar{c}}(g g)$ & 39 & 6.4 & 0.25 & 0.45 & 0.07 & 0.022 & $1.2 \mathrm{E}-3$ & 0.18 \\
$R_{c \bar{c}}(b \bar{b})$ & 0.21 & 0.66 & 0.1 & 0.03 & 0.008 & 0.002 & 0.0001 & 0.0013 \\
\hline$R_{\tau \tau}(g g)$ & 2421 & 833 & $.001-62$ & 10 & 3.12 & 0.81 & 0.07 & 0.58 \\
$R_{\tau \tau}(b \bar{b})$ & 13.4 & 87 & $4 \mathrm{E}-4-24$ & $1 \mathrm{E}-4-13.6$ & $1 \mathrm{E}-4-3.7$ & 3.2 & .15 & .184 \\
\hline$R_{\mu \mu}(g g)$ & 8.8 & 3.0 & 0.22 & 0.035 & .011 & $2.8 \mathrm{E}-3$ & $2.5 \mathrm{E}-4$ & $2.1 \mathrm{E}-3$ \\
$R_{\mu \mu}(b \bar{b})$ & 0.05 & 0.31 & 0.1 & 0.05 & 0.013 & 0.011 & $5 \mathrm{E}-4$ & $6 \mathrm{E}-4$ \\
\hline$R_{\gamma \gamma}(g g)$ & 0.3 & 0.2 & $4 \mathrm{E}-3-0.11$ & 0.042 & 0.058 & 0.017 & $8.4 \mathrm{E}-3$ & 0.035 \\
$R_{\gamma \gamma}(b \bar{b})$ & $2 \mathrm{E}-3$ & 0.01 & $2 \mathrm{E}-3-0.058$ & $1 \mathrm{E}-4-0.06$ & 0.04 & $6 \mathrm{E}-3$ & $3 \mathrm{E}-4$ & $2.1 \mathrm{E}-3$ \\
\hline$R_{g g}(g g)$ & 305 & 46.3 & $2.5 \mathrm{E}-3-1.7$ & 0.95 & 0.22 & 0.1 & $6 \mathrm{E}-3$ & 0.97 \\
$R_{g g}(b \bar{b})$ & 1.7 & 4.8 & 0.66 & 0.15 & 0.025 & 0.014 & $7 \mathrm{E}-4$ & $7 \mathrm{E}-3$ \\
\hline
\end{tabular}

Table 4: Production rates(cross section $\times$ branching ratio) $(\mathrm{fb})$ of $A_{1}$ in various decay channels at $13 \mathrm{TeV}$ LHC energy for case (B).

\begin{tabular}{|c|cccc|}
\hline Rates & \multicolumn{5}{|c|}{$m_{H_{2}}(\mathrm{GeV})$} \\
& 137 & 200 & 300 & 400 \\
\hline$R_{b \bar{b}}(g g)$ & 32 & $1.1-7.68$ & $2.5 \mathrm{E}-4-0.18$ & $1.6 \mathrm{E}-4-0.05$ \\
$R_{b \bar{b}}(b \bar{b})$ & 78 & $7.4 \mathrm{E}-3-1.5$ & $9.1 \mathrm{E}-4-0.066$ & $1.5 \mathrm{E}-3$ \\
\hline$R_{\tilde{\chi}_{1}^{0} \tilde{\chi}_{1}^{0}}(g g)$ & - & $30-58.32$ & 5.0 & $7 \mathrm{E}-4$ \\
$R_{\tilde{\chi}_{1}^{0} \tilde{\chi}_{1}^{0}}(b \bar{b})$ & - & $0.19-11.51$ & $3.5 \mathrm{E}-4-1.8$ & $1 \mathrm{E}-4$ \\
\hline$R_{W W}(g g)$ & 0.1 & $19-149$ & 3.1 & $1 \mathrm{E}-3-13$ \\
$R_{W W}(b \bar{b})$ & 0.24 & $0.98-3.7$ & $5.6 \mathrm{E}-4-0.28$ & $2.2 \mathrm{E}-4-0.26$ \\
\hline$R_{A_{1} A_{1}}(g g)$ & - & 602 & 37 & $0.35-24$ \\
$R_{A_{1} A_{1}}(b \bar{b})$ & - & 4 & 3 & $4.5 \mathrm{E}-2-0.95$ \\
\hline$R_{H_{1} H_{1}}(g g)$ & 0 & 0 & 2.8 & $3.6 \mathrm{E}-4-7.5$ \\
$R_{H_{1} H_{1}}(b \bar{b})$ & - & - & 0.4 & 0.15 \\
\hline
\end{tabular}

Table 5: Production rates(cross section $\times$ branching ratio)(fb) of $\mathrm{H}_{2}$ at $13 \mathrm{TeV}$ for case (B) 


\section{Higgs signal}

The discovery potential of the non-SM-like Higgs boson depends on the underlying production mechanism along with its subsequent decay spectrum and the resulting rate. Already many studies exist in the literature presenting the detection prospect of finding the non SM Higgs bosons in the NMSSM exploiting, in particular popular $b \bar{b}, \tau \tau$ modes producing Higgs bosons directly or indirectly via Higgs to Higgs decays (for a review and details see Refs. 14, 85, 102 and references therein). However, here we try to emphasize the detection prospect of the non-SM-like Higgs signal via enhanced rate in the $\gamma \gamma$ final state and as well as the other final states such as gg, cc.

As we have noticed in the previous sections, the $\mathrm{BR}$ in $\gamma \gamma$ mode of $\mathrm{A}_{1}$ and $H_{1}$ can be very large, in particular for $A_{1}$, it is closer to $90-100 \%$ in a certain region of parameter space in the scenario case(B), see Fig, 9 18, 23, 24, 103 and similarly the BR of SM-like Higgs boson of mass $125 \mathrm{GeV}$ in the di-photon is also enhanced in comparison to SM Higgs boson [104]. The BR of $\mathrm{H}_{1} \rightarrow \gamma \gamma$ is also not negligible and is observed to be around $2-3 \%$ for $m_{H_{1}}$ masses $\sim 60 \mathrm{GeV}$ or above as seen in the Fig 7 and the Table 2 105]. For example, for $m_{H_{1}} \sim 68 \mathrm{GeV}$, (see Table 2), at $13 \mathrm{TeV}$ LHC energy, one can have maximum about few thousands events in $\gamma \gamma$ channel for the integrated luminosity $100 \mathrm{fb}^{-1}$ For the same scenario, in case of $\mathrm{A}_{1}$, for 60 GeV mass, one can expect about fewer event $\sim 10-15$ (see Table 3 for the same integrated luminosity. Here, the large $\mathrm{BR}$ of $\mathrm{A}_{1}$ into two photons channel does not yield a large rate because of suppressed production cross section. As is well known, looking for a signal in the di-photon final state is very promising as photon is a very clean object to tag experimentally. It is remarkable that neither in the SM nor in the MSSM, the decay rates of the Higgs bosons in the di-photon channel is comparable to what is in the NMSSM. For instance, in Refs. 23, 103] it is shown that for the $\mathrm{H}_{1}$, the relative signal rates i.e production cross sections times $\mathrm{BR}\left(\mathrm{H}_{1} \rightarrow \gamma \gamma\right)$ are almost larger by almost a factor of 5-7 what is predicted by the SM for $\mathrm{m}_{\mathrm{H}_{1}} \lesssim 100 \mathrm{GeV}$ and similar pattern is also observed even for $\mathrm{m}_{\mathrm{H}_{1}} \gtrsim 100 \mathrm{GeV}$ case [106]. In this current study we focus on the di-photon channel for a very low mass region $(<125 \mathrm{GeV})$ of non SM-like Higgs bosons which are accessible at the current LHC experiments. After performing a very systematic scanning of the parameter space we present the minimum and maximum rates of di-photon production for a given mass of non-SM-like Higgs. In addition we also discussed the variation of the BR of the di-photon channel for this mass range along with other decay channels. It is worth to have this information in order to estimate the sensitivity of non SM-like Higgs production for a given integrated luminosity. Evidently, this diphoton channel appears to be a smoking gun signal to look for light neutral non SM Higgs bosons. Although some simple parton-level analyses were already carried out in the past to explore the detection possibility in this di-photon channel [106, 107], more detail analysis are required in the present context. The estimation of the corresponding SM background in this di-photon channel is also a challenging task, in particular, for the low mass Higgs bosons. We postpone this analysis to a future work [108.

Furthermore, for Higgs decays to two gluons, $\mathrm{H}_{1} \rightarrow$ gg with a branching ratio of about $50 \%$ or more, a reasonable number of events in this channel are expected for the $100 \mathrm{fb}^{-1}$ integrated luminosity (see Fig.7 and Table 2), in particular for the mass range $\sim 60-100 \mathrm{GeV}$, this channel yields a huge number of events $(\mathcal{O}(1000))$ even for an 1 integrated luminosity as low as $10 \mathrm{fb}^{-1}$. Clearly, this gg final state provides an alternative option to detect the NMSSM light neutral Higgs bosons at the LHC by probing the invariant mass of a pair of jets, which 
is not a easy task due to the presence of enormous QCD background. Perhaps, in the $\mathrm{H}_{1}+1$ jet final state, where an extra jet comes from radiation followed by $\mathrm{H}_{1} \rightarrow$ gg decay, one can tag two jets to reconstruct the Higgs mass using the jet substructure method which is a very efficient technique [109 to probe boosted objects. Of course, the feasibility of this channel can be understood only after carrying out a detailed simulation including QCD background. Possibly, this $g g$ channel provides a direct opportunity for a direct measurement of effective Higgs coupling to gluons directly [110]. In addition, the decays of the Higgs bosons to SUSY particles, such as, $\phi \rightarrow \tilde{\chi}_{1}^{0} \tilde{\chi}_{2}^{0}$, if are kienamtically allowed and have a reasonable BR, can also lead to interesting signals, particularly for leptonic decays of sparticles. Essentially, sparticle decay channels open up for values of $m_{H_{1}} \sim 200 \mathrm{GeV}$. These decay channel $\phi \tilde{\chi}_{1}^{0} \tilde{\chi}_{1}^{0}$ may also contribute to the invisible width of the Higgs boson. Finally, Higgs to Higgs decay channels (Eq.3.26) give rise to many more detectable final states depending on the decays of $H_{1}$ and $A_{1}$. A detail investigation of these channels and measurements of triple Higgs couplings may reveal the model structure of the NMSSM. In Table 6, for the sake of illustration we present for few cases some of the relevant parameters where the rates are reasonably large for a given Higgs boson mass and decay channel.

\begin{tabular}{|c|c|c|c|c|}
\hline & $\mathrm{P} 1$ & $\mathrm{P} 2$ & P3 & $\mathrm{P} 4$ \\
\hline Case & $\mathrm{A}$ & $\mathrm{A}$ & B & B \\
\hline $\mathrm{M}_{\mathrm{H}_{\mathrm{i}}}(\mathrm{GeV})$ & $13(\mathrm{i}=1)$ & $67(\mathrm{i}=1)$ & $200(\mathrm{i}=2)$ & $400(\mathrm{i}=2)$ \\
\hline$R_{X X}(g g)(f b)$ & $144927(b \bar{b}), 13838(\tau \tau)$ & $93(\gamma \gamma), 2881(g g), 3737(c \bar{c})$ & $602\left(A_{1} A_{1}\right), 149(W W)$ & $24\left(A_{1} A_{1}\right), 13.03(W W)$ \\
\hline$\lambda, \kappa$ & $0.363,0,148$ & $0.325,0.167$ & $0.544,0.265$ & $0.64,0.317$ \\
\hline $\tan \beta \mu$ & $9.27,165.45$ & $8.36,229.02$ & 2.45, 192.7 & $2.33,401.30$ \\
\hline$A_{\lambda}, A_{\kappa}$ & $1440.7,-273.95$ & $1658.38,-450.35$ & $508.06,3.69$ & $530.27,-35.35$ \\
\hline$M_{Q_{3}}, M_{U_{3}}$ & $2828.25,358.58$ & $1847.6, \quad 2950.2$ & $2593,1686.8$ & $1138.32, \quad 1427.90$ \\
\hline$A_{t}$ & -2574.43 & -2779.78 & -3000.0 & -3016.5 \\
\hline \multirow[t]{2}{*}{$M_{1}, M_{2}$} & $89.18,335.99$ & $75.9,414.18$ & $92.89,816.69$ & $80.6, \quad 114.3$ \\
\hline & $\mathrm{P} 5$ & $\mathrm{P} 6$ & $\mathrm{P} 7$ & $\mathrm{P} 8$ \\
\hline Case & A & $\mathrm{B}$ & $\mathrm{A}$ & $\mathrm{B}$ \\
\hline $\mathrm{M}_{\mathrm{A}_{1}}(\mathrm{GeV})$ & 22 & 60 & 100 & 249 \\
\hline$R_{X X}(g g)(f b)$ & $72336(b \bar{b}), 5939(\tau \tau)$ & $0.112(\gamma \gamma)$ & $226(b \bar{b}), 23(\tau \tau)$ & $0.035(\gamma \gamma)$ \\
\hline$\lambda, \kappa$ & $0.368,0.103$ & $0.328,0.434$ & $0.677,0.182$ & $0.499,0.476$ \\
\hline $\tan \beta \mu$ & $8.56,154.74$ & $5.61,128.7$ & $7.66,193.0$ & $1.55,1859.0$ \\
\hline$A_{\lambda}, A_{\kappa}$ & $1432.96,-0.486$ & $553.3,-1.53$ & $1464.15,-81.22$ & $872.53,-13.41$ \\
\hline$M_{Q_{3}}, M_{U_{3}}$ & $2237.9,829.6$ & $2744.15, \quad 1441.90$ & $1962.45,2666.53$ & $1289.96,1165.72$ \\
\hline$A_{t}, A_{b}$ & -2516.9 & -3914.36 & 1850.3 & -1617 \\
\hline$M_{1}, M_{2}$ & $396.07,319.15$ & $98.19,503.6$ & $410.8,160.43$ & $56.2, \quad 574.26$ \\
\hline
\end{tabular}

Table 6: Parameter sets(P1 - P8) for a given Higgs boson mass and decay channel for both the cases $\mathrm{A}$ and $\mathrm{B}$ corresponding to the rates $R_{X X}(\mathrm{in} \mathrm{fb})$. These points $\mathrm{P} 1-\mathrm{P} 8$ are selected from Tabe 2-5. 


\section{Summary}

The recently discovered Higgs particle can be accommodated in the framework of the NMSSM easily without much adjusting the input parameters unlike in the case of the MSSM. Scanning the parameter space taking into account all constraints, we found the scenario where one of the Higgs bosons is SM-like and, some of the singlet like neutral Higgs bosons can be rather very light with suppressed couplings to fermions and the gauge bosons. In this work we focus our attention only to light neutral two CP even Higgs bosons and one CP odd Higgs boson out of which one the CP even Higgs bosons is SM-like. We discuss the masses and the couplings of the non-SM-like light neutral Higgs bosons and their BRs in various decay channels which predict the Higgs phenomenology at colliders. We present the BRs of the nonSM-like Higgs bosons in all accessible decay channels in reference to the allowed parameter space. We present the ranges of over all rates of production for a given non SM Higgs mass and for a given decay channel in the allowed region of parameter space, which demonstrate the potential final states that can be probed in the search for non SM signal at the LHC. We found some of the characteristic decay channels which are less dominant in the the SM and in the MSSM could play important roles in such searches. For example, in certain region of the parameter space, a large BR of Higgs decay to two photons is found to be quite interesting and can provide not only a characteristic robust signal of the NMSSM, but can also help to discriminate the NMSSM from the MSSM. Although this observation is also made in previous analyses, as discussed in sec. 5 , but here we present the rates in this di-photon channel more systematic way for various lower masses of Higgs bosons corresponding to the center of mass energy at $13 \mathrm{TeV}$. These information may be very useful in searching for the non SM-like Higgs bosons at the LHC. Higgs decay to two gluons also turn out to be an interesting mode which one can exploit to study the the NMSSM Higgs sector, though care must be taken to deal with an enormous QCD background. Moreover, further studies of Higgs to Higgs decays via triple Higgs bosons couplings and probing the associated signals in various final states could uncover the underlying dynamics of the model.

\section{Acknowledgement}

The authors are grateful to Asesh Krishna Datta for his careful reading of the manuscript and many valuable suggestions. They are also thankful to Debottom Das and Abhishek Iyer for discussion.

\section{References}

[1] CMS Collaboration, S. Chatrchyan et al., "Observation of a new boson at a mass of $125 \mathrm{GeV}$ with the CMS experiment at the LHC," Phys.Lett. B716 (2012) 30-61, arXiv:1207.7235 [hep-ex].

[2] ATLAS Collaboration, G. Aad et al., "Observation of a new particle in the search for the Standard Model Higgs boson with the ATLAS detector at the LHC," Phys.Lett. B716 (2012) 1-29, arXiv: 1207.7214 [hep-ex]. 
[3] CMS Collaboration Collaboration, V. Khachatryan et al., "Precise determination of the mass of the Higgs boson and tests of compatibility of its couplings with the standard model predictions using proton collisions at 7 and $8 \mathrm{TeV}$," arXiv:1412.8662 [hep-ex].

[4] F. Barreiro, "Higgs boson production and couplings with the ATLAS detector,".

[5] ATLAS, CMS Collaboration, G. Aad et al., "Combined Measurement of the Higgs Boson Mass in $p p$ Collisions at $\sqrt{s}=7$ and $8 \mathrm{TeV}$ with the ATLAS and CMS Experiments," Phys. Rev. Lett. 114 (2015) 191803, arXiv:1503.07589 [hep-ex].

[6] L. J. Hall, D. Pinner, and J. T. Ruderman, "A Natural SUSY Higgs Near 126 GeV," JHEP 04 (2012) 131, arXiv:1112.2703 [hep-ph].

[7] A. Arbey, M. Battaglia, A. Djouadi, F. Mahmoudi, and J. Quevillon, "Implications of a 125 GeV Higgs for supersymmetric models," Phys. Lett. B708 (2012) 162-169, arXiv:1112.3028 [hep-ph].

[8] R. Barbieri and G. F. Giudice, "Upper Bounds on Supersymmetric Particle Masses," Nucl. Phys. B306 (1988) 63.

[9] R. Kitano and Y. Nomura, "Supersymmetry, naturalness, and signatures at the LHC," Phys. Rev. D73 (2006) 095004, arXiv:hep-ph/0602096 [hep-ph].

[10] J. R. Ellis, J. Gunion, H. E. Haber, L. Roszkowski, and F. Zwirner, "Higgs Bosons in a Nonminimal Supersymmetric Model," Phys.Rev. D39 (1989) 844.

[11] M. Drees, "Supersymmetric Models with Extended Higgs Sector," Int.J.Mod.Phys. A4 (1989) 3635 .

[12] V. Barger, P. Langacker, H.-S. Lee, and G. Shaughnessy, "Higgs Sector in Extensions of the MSSM," Phys.Rev. D73 (2006) 115010, arXiv:hep-ph/0603247| [hep-ph].

[13] U. Ellwanger, C. Hugonie, and A. M. Teixeira, "The Next-to-Minimal Supersymmetric Standard Model," Phys.Rept. 496 (2010) 1-77, arXiv:0910.1785 [hep-ph].

[14] U. Ellwanger, "Higgs Bosons in the Next-to-Minimal Supersymmetric Standard Model at the LHC," Eur.Phys.J. C71 (2011) 1782, arXiv:1108.0157 [hep-ph].

[15] S. King, M. Muhlleitner, and R. Nevzorov, "NMSSM Higgs Benchmarks Near 125 GeV," Nucl.Phys. B860 (2012) 207-244, arXiv:1201.2671 [hep-ph].

[16] D. A. Vasquez, G. Belanger, C. Boehm, J. Da Silva, P. Richardson, et al., "The 125 GeV Higgs in the NMSSM in light of LHC results and astrophysics constraints," \begin{tabular}{|l|l|l|}
\hline Phys.Rev. D86 (2012) 035023, arXiv:1203.3446 [hep-ph].
\end{tabular}

[17] S. King, M. Mhlleitner, R. Nevzorov, and K. Walz, "Natural NMSSM Higgs Bosons," Nucl.Phys. B870 (2013) 323-352, arXiv:1211.5074 [hep-ph].

[18] N. D. Christensen, T. Han, Z. Liu, and S. Su, "Low-Mass Higgs Bosons in the NMSSM and Their LHC Implications," JHEP 1308 (2013) 019, arXiv:1303.2113. 
[19] J. Cao, F. Ding, C. Han, J. M. Yang, and J. Zhu, "A light Higgs scalar in the NMSSM confronted with the latest LHC Higgs data," JHEP 1311 (2013) 018 , arXiv: 1309.4939 [hep-ph].

[20] Z. Kang, J. Li, and T. Li, "On Naturalness of the MSSM and NMSSM," JHEP 11 (2012) 024, arXiv:1201.5305 [hep-ph].

[21] CMS Collaboration, S. Chatrchyan et al., "Search for a light pseudoscalar Higgs boson in the dimuon decay channel in $p p$ collisions at $\sqrt{s}=7 \mathrm{TeV}$," Phys. Rev. Lett. 109 (2012) 121801, arXiv:1206.6326 [hep-ex].

[22] ATLAS Collaboration, G. Aad et al., "Search for Higgs bosons decaying to aa in the $\mu \mu \tau \tau$ final state in $p p$ collisions at $\sqrt{s}=8 \mathrm{TeV}$ with the ATLAS experiment," arXiv:1505.01609 [hep-ex].

[23] U. Ellwanger, "Enhanced di-photon Higgs signal in the Next-to-Minimal Supersymmetric Standard Model," Phys.Lett. B698 (2011) 293-296, arXiv:1012.1201 [hep-ph].

[24] S. King, M. Mhlleitner, R. Nevzorov, and K. Walz, "Discovery Prospects for NMSSM Higgs Bosons at the High-Energy Large Hadron Collider," Phys.Rev. D90 no. 9, (2014) 095014, arXiv: 1408.1120 [hep-ph].

[25] S. Liebler, "Neutral Higgs production at proton colliders in the CP-conserving NMSSM," Eur. Phys. J. C75 no. 5, (2015) 210, arXiv:1502.07972 [hep-ph].

[26] R. Peccei and H. R. Quinn, "CP Conservation in the Presence of Instantons," Phys.Rev.Lett. 38 (1977) 1440-1443.

[27] F. Franke and H. Fraas, "Neutralinos and Higgs bosons in the next-to-minimal supersymmetric standard model," Int.J.Mod.Phys. A12 (1997) 479-534, arXiv:hep-ph/9512366 [hep-ph].

[28] D. Miller, R. Nevzorov, and P. Zerwas, "The Higgs sector of the next-to-minimal supersymmetric standard model," Nucl.Phys. B681 (2004) 3-30, arXiv:hep-ph/0304049 [hep-ph].

[29] ALEPH, DELPHI, L3, OPAL, LEP Working Group for Higgs Boson Searches Collaboration, S. Schael et al., "Search for neutral MSSM Higgs bosons at LEP," Eur.Phys.J. C47 (2006) 547-587, arXiv:hep-ex/0602042 [hep-ex].

[30] U. Ellwanger, M. Rausch de Traubenberg, and C. A. Savoy, "Particle spectrum in supersymmetric models with a gauge singlet," Phys.Lett. B315 (1993) 331-337, arXiv:hep-ph/9307322 [hep-ph].

[31] S. King and P. White, "Resolving the constrained minimal and next-to-minimal supersymmetric standard models," Phys.Rev. D52 (1995) 4183-4216, arXiv:hep-ph/9505326 [hep-ph].

[32] U. Ellwanger, "Radiative corrections to the neutral Higgs spectrum in supersymmetry with a gauge singlet," Phys.Lett. B303 (1993) 271-276, arXiv:hep-ph/9302224 [hep-ph]. 
[33] T. Elliott, S. King, and P. White, "Radiative corrections to Higgs boson masses in the next-to-minimal supersymmetric Standard Model," Phys.Rev. D49 (1994) 2435-2456, arXiv:hep-ph/9308309 [hep-ph].

[34] T. Elliott, S. King, and P. White, "Supersymmetric Higgs bosons at the limit," Phys.Lett. B305 (1993) 71-77, arXiv:hep-ph/9302202 [hep-ph].

[35] P. Pandita, "Radiative corrections to the scalar Higgs masses in a nonminimal supersymmetric Standard Model," Z.Phys. C59 (1993) 575-584.

[36] P. Pandita, "One loop radiative corrections to the lightest Higgs scalar mass in nonminimal supersymmetric Standard Model," Phys.Lett. B318 (1993) 338-346.

[37] U. Ellwanger and C. Hugonie, "Yukawa induced radiative corrections to the lightest Higgs boson mass in the NMSSM," Phys.Lett. B623 (2005) 93-103, arXiv:hep-ph/0504269 [hep-ph].

[38] G. Degrassi and P. Slavich, "On the radiative corrections to the neutral Higgs boson masses in the NMSSM," Nucl.Phys. B825 (2010) 119-150, arXiv:0907.4682 [hep-ph].

[39] K. Ender, T. Graf, M. Muhlleitner, and H. Rzehak, "Analysis of the NMSSM Higgs Boson Masses at One-Loop Level," Phys.Rev. D85 (2012) 075024, arXiv:1111.4952 [hep-ph].

[40] M. D. Goodsell, K. Nickel, and F. Staub, "On the two-loop corrections to the Higgs masses in the NMSSM," arXiv:1411.4665 [hep-ph].

[41] M. Mhlleitner, D. T. Nhung, H. Rzehak, and K. Walz, "Two-loop contributions of the order $\mathcal{O}\left(\alpha_{t} \alpha_{s}\right)$ to the masses of the Higgs bosons in the CP-violating NMSSM," JHEP 05 (2015) 128, arXiv:1412.0918 [hep-ph].

[42] U. Ellwanger and C. Hugonie, "The Upper bound on the lightest Higgs mass in the NMSSM revisited," Mod.Phys.Lett. A22 (2007) 1581-1590, arXiv:hep-ph/0612133 [hep-ph].

[43] R. Barbieri, L. J. Hall, Y. Nomura, and V. S. Rychkov, "Supersymmetry without a Light Higgs Boson," Phys.Rev. D75 (2007) 035007, arXiv:hep-ph/0607332 [hep-ph].

[44] J. Beuria, A. Chatterjee, A. Datta, and S. K. Rai, "Two Light Stops in the NMSSM and the LHC," arXiv:1505.00604 [hep-ph].

[45] F. Staub, P. Athron, U. Ellwanger, R. Grober, M. Muhlleitner, P. Slavich, and A. Voigt, "Higgs mass predictions of public NMSSM spectrum generators," arXiv:1507.05093 [hep-ph].

[46] Particle Data Group Collaboration, K. Olive et al., "Review of Particle Physics," Chin.Phys. C38 (2014) 090001.

[47] U. Ellwanger and C. Hugonie, "The semi-constrained NMSSM satisfying bounds from the LHC, LUX and Planck," JHEP 1408 (2014) 046, arXiv:1405.6647| [hep-ph]. 
[48] U. Ellwanger, J. F. Gunion, and C. Hugonie, "NMHDECAY: A Fortran code for the Higgs masses, couplings and decay widths in the NMSSM," JHEP 0502 (2005) 066. arXiv:hep-ph/0406215 [hep-ph].

[49] U. Ellwanger and C. Hugonie, "NMHDECAY 2.0: An Updated program for sparticle masses, Higgs masses, couplings and decay widths in the NMSSM," Comput.Phys.Commun. 175 (2006) 290-303, arXiv:hep-ph/0508022 [hep-ph].

[50] K. Agashe, Y. Cui, and R. Franceschini, "Natural Islands for a $125 \mathrm{GeV}$ Higgs in the scale-invariant NMSSM," JHEP 02 (2013) 031, arXiv:1209.2115 [hep-ph].

[51] J. Cao, Z. Heng, J. M. Yang, and J. Zhu, "Status of low energy SUSY models confronted with the LHC $125 \mathrm{GeV}$ Higgs data," JHEP 10 (2012) 079 , arXiv:1207.3698 [hep-ph].

[52] U. Ellwanger and C. Hugonie, "NMSPEC: A Fortran code for the sparticle and Higgs masses in the NMSSM with GUT scale boundary conditions," Comput.Phys.Commun. 177 (2007) 399-407, arXiv:hep-ph/0612134 [hep-ph].

[53] Planck Collaboration, P. Ade et al., "Planck 2013 results. XVI. Cosmological parameters," Astron.Astrophys. 571 (2014) A16, arXiv:1303.5076 [astro-ph.C0].

[54] Muon g-2 Collaboration, G. Bennett et al., "Measurement of the negative muon anomalous magnetic moment to 0.7 ppm," Phys.Rev.Lett. 92 (2004) 161802, arXiv:hep-ex/0401008 [hep-ex].

[55] G. Belanger, F. Boudjema, A. Pukhov, and A. Semenov, "micrOMEGAs 3: A program for calculating dark matter observables," Comput. Phys. Commun. 185 (2014) 960-985, arXiv:1305.0237 [hep-ph].

[56] G. Belanger, F. Boudjema, C. Hugonie, A. Pukhov, and A. Semenov, "Relic density of dark matter in the NMSSM," JCAP 0509 (2005) 001, arXiv: hep-ph/0505142 [hep-ph].

[57] D. Albornoz Vasquez, G. Belanger, J. Billard, and F. Mayet, "Probing neutralino dark matter in the MSSM \& the NMSSM with directional detection," Phys.Rev. D85 \begin{tabular}{|l|l|}
\hline (2012) 055023, & arXiv:1201.6150 [hep-ph].
\end{tabular}

[58] J. Kozaczuk and S. Profumo, "Light NMSSM neutralino dark matter in the wake of CDMS II and a 126 GeV Higgs boson," Phys.Rev. D89 no. 9, (2014) 095012, arXiv:1308.5705 [hep-ph].

[59] D. E. Lopez-Fogliani, "Light Higgs and neutralino dark matter in the NMSSM," J.Phys.Conf.Ser. 384 (2012) 012014.

[60] J.-J. Cao, K.-i. Hikasa, W. Wang, J. M. Yang, K.-i. Hikasa, et al., "Light dark matter in NMSSM and implication on Higgs phenomenology," Phys.Lett. B703 (2011) 292-297, arXiv:1104.1754 [hep-ph].

[61] D. Das and U. Ellwanger, "Light dark matter in the NMSSM: upper bounds on direct detection cross sections," JHEP 1009 (2010) 085, arXiv: 1007.1151 [hep-ph]. 
[62] C. Hugonie, G. Belanger, and A. Pukhov, "Dark matter in the constrained NMSSM," JCAP 0711 (2007) 009, arXiv:0707.0628 [hep-ph].

[63] D. E. Lopez-Fogliani, "Neutralino Dark Matter in the NMSSM," J.Phys. A40 (2007) 6889-6894, arXiv:hep-ph/0703181 [HEP-PH].

[64] D. Cerdeno, E. Gabrielli, D. Lopez-Fogliani, C. Munoz, and A. Teixeira, "Phenomenological viability of neutralino dark matter in the NMSSM," JCAP 0706 (2007) 008, arXiv:hep-ph/0701271 [HEP-PH]

[65] J. F. Gunion, D. Hooper, and B. McElrath, "Light neutralino dark matter in the NMSSM," Phys.Rev. D73 (2006) 015011, arXiv:hep-ph/0509024 [hep-ph].

[66] R. Enberg, S. Munir, C. Prez de los Heros, and D. Werder, "Prospects for higgsino-singlino dark matter detection at IceCube and PINGU," arXiv:1506.05714 [hep-ph].

[67] F. Jegerlehner, "Essentials of the Muon g-2," Acta Phys.Polon. B38 (2007) 3021, arXiv:hep-ph/0703125 [hep-ph].

[68] M. Endo, K. Hamaguchi, S. Iwamoto, and T. Yoshinaga, "Muon g-2 vs LHC in

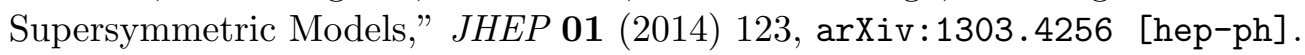

[69] T. Moroi, "The Muon anomalous magnetic dipole moment in the minimal supersymmetric standard model," Phys. Rev. D53 (1996) 6565-6575, arXiv:hep-ph/9512396 [hep-ph]. [Erratum: Phys. Rev.D56,4424(1997)].

[70] C. Gnendiger, D. Stckinger, and H. Stckinger-Kim, "The electroweak contributions to $(g-2) \_\mu$ after the Higgs boson mass measurement," Phys. Rev. D88 (2013) 053005 , arXiv:1306.5546 [hep-ph].

[71] H. Fargnoli, C. Gnendiger, S. Paehr, D. Stckinger, and H. Stckinger-Kim, "Two-loop corrections to the muon magnetic moment from fermion/sfermion loops in the MSSM: detailed results," JHEP 02 (2014) 070, arXiv:1311.1775 [hep-ph].

[72] F. Domingo and U. Ellwanger, "Constraints from the Muon g-2 on the Parameter Space of the NMSSM," JHEP 0807 (2008) 079, arXiv:0806.0733 [hep-ph].

[73] M. Krawczyk, "Precision muon g-2 results and light Higgs bosons in the 2HDM(II)," Acta Phys.Polon. B33 (2002) 2621-2634, arXiv:hep-ph/0208076 [hep-ph]

[74] S. Heinemeyer, D. Stockinger, and G. Weiglein, "Electroweak and supersymmetric two-loop corrections to (g-2)(mu)," Nucl.Phys. B699 (2004) 103-123, arXiv:hep-ph/0405255 [hep-ph].

[75] LHC Higgs Cross Section Working Group Collaboration, S. Heinemeyer et al., "Handbook of LHC Higgs Cross Sections: 3. Higgs Properties," arXiv:1307.1347 [hep-ph].

[76] R. Dermisek and J. F. Gunion, "Many Light Higgs Bosons in the NMSSM," Phys.Rev. D79 (2009) 055014, arXiv:0811.3537 [hep-ph]. 
[77] J. Louis and L. Zarate, "Hiding the little hierarchy problem in the NMSSM," JHEP 08 (2015) 062, arXiv: 1506.01616 [hep-ph].

[78] D. T. Nhung, M. Muhlleitner, J. Streicher, and K. Walz, "Higher Order Corrections to the Trilinear Higgs Self-Couplings in the Real NMSSM," JHEP 1311 (2013) 181. arXiv:1306.3926 [hep-ph].

[79] M. Mhlleitner, D. T. Nhung, and H. Ziesche, "The order $\mathcal{O}\left(\alpha_{t} \alpha_{s}\right)$ corrections to the trilinear Higgs self-couplings in the complex NMSSM," JHEP 12 (2015) 034, arXiv: 1506.03321 [hep-ph].

[80] M. Badziak, M. Olechowski, and S. Pokorski, "New Regions in the NMSSM with a 125 GeV Higgs," JHEP 1306 (2013) 043, arXiv: 1304.5437 [hep-ph].

[81] G. Belanger, V. Bizouard, and G. Chalons, "Boosting Higgs boson decays into gamma and a Z in the NMSSM," Phys. Rev. D89 no. 9, (2014) 095023, arXiv:1402.3522 [hep-ph].

[82] S. Munir, L. Roszkowski, and S. Trojanowski, "Simultaneous enhancement in $\gamma \gamma, b \bar{b}$ and $\tau^{+} \tau^{-}$rates in the NMSSM with nearly degenerate scalar and pseudoscalar Higgs bosons," Phys. Rev. D88 no. 5, (2013) 055017, arXiv:1305.0591| [hep-ph].

[83] M. Zhang, "The Heavy Higgs Decay Effect and Its Impact on Higgs Data Fitting in NMSSM," arXiv:1505.05636 [hep-ph].

[84] B. Ananthanarayan, J. Lahiri, and P. Pandita, "Invisible decays of the heavier Higgs boson in the minimal supersymmetric standard model," Phys. Rev. D91 (2015) 115025, arXiv:1507.01747 [hep-ph].

[85] N.-E. Bomark, S. Moretti, S. Munir, and L. Roszkowski, "A light NMSSM pseudoscalar Higgs boson at the LHC redux," JHEP 1502 (2015) 044, arXiv: 1409.8393 [hep-ph].

[86] R. V. Harlander, "Virtual corrections to g g - i H to two loops in the heavy top limit," Phys. Lett. B492 (2000) 74-80, arXiv:hep-ph/0007289 [hep-ph].

[87] S. Catani, D. de Florian, and M. Grazzini, "Higgs production in hadron collisions: Soft and virtual QCD corrections at NNLO," JHEP 05 (2001) 025, arXiv:hep-ph/0102227 [hep-ph].

[88] R. V. Harlander and W. B. Kilgore, "Soft and virtual corrections to proton proton -i $\mathrm{H}+\mathrm{x}$ at NNLO," Phys. Rev. D64 (2001) 013015, arXiv:hep-ph/0102241 [hep-ph].

[89] R. V. Harlander and W. B. Kilgore, "Next-to-next-to-leading order Higgs production at hadron colliders," Phys. Rev. Lett. 88 (2002) 201801, arXiv:hep-ph/0201206 [hep-ph].

[90] C. Anastasiou and K. Melnikov, "Higgs boson production at hadron colliders in NNLO QCD," Nucl. Phys. B646 (2002) 220-256, arXiv:hep-ph/0207004 [hep-ph].

[91] V. Ravindran, J. Smith, and W. L. van Neerven, "NNLO corrections to the total cross-section for Higgs boson production in hadron hadron collisions," Nucl. Phys. B665 (2003) 325-366, arXiv:hep-ph/0302135 [hep-ph]. 
[92] J. Blumlein and V. Ravindran, "Mellin moments of the next-to-next-to leading order coefficient functions for the Drell-Yan process and hadronic Higgs-boson production," \begin{tabular}{|l|l|l|}
\hline Nucl. Phys. B716 (2005) 128-172, arXiv:hep-ph/0501178 [hep-ph]. \\
\hline
\end{tabular}

[93] R. V. Harlander, S. Liebler, and H. Mantler, "SusHi: A program for the calculation of Higgs production in gluon fusion and bottom-quark annihilation in the Standard Model and the MSSM," Comput.Phys.Commun. 184 (2013) 1605-1617, arXiv:1212.3249 [hep-ph].

[94] A. Djouadi, "The Anatomy of electro-weak symmetry breaking. II. The Higgs bosons in the minimal supersymmetric model," Phys. Rept. 459 (2008) 1-241, arXiv:hep-ph/0503173 [hep-ph].

[95] G. Degrassi and P. Slavich, "NLO QCD bottom corrections to Higgs boson production in the MSSM," JHEP 11 (2010) 044, arXiv:1007.3465 [hep-ph].

[96] G. Degrassi, S. Di Vita, and P. Slavich, "NLO QCD corrections to pseudoscalar Higgs production in the MSSM," JHEP 08 (2011) 128, arXiv:1107.0914 [hep-ph].

[97] G. Degrassi, S. Di Vita, and P. Slavich, "On the NLO QCD Corrections to the Production of the Heaviest Neutral Higgs Scalar in the MSSM," Eur. Phys. J. C72 (2012) 2032, arXiv:1204.1016 [hep-ph].

[98] LHC Higgs Cross Section Working Group Collaboration, S. Dittmaier et al., "Handbook of LHC Higgs Cross Sections: 1. Inclusive Observables," arXiv:1101.0593 [hep-ph].

[99] S. Dittmaier, . Kramer, Michael, and M. Spira, "Higgs radiation off bottom quarks at the Tevatron and the CERN LHC," Phys.Rev. D70 (2004) 074010, arXiv:hep-ph/0309204 [hep-ph].

[100] F. Maltoni, Z. Sullivan, and S. Willenbrock, "Higgs-boson production via bottom-quark fusion," Phys.Rev. D67 (2003) 093005, arXiv:hep-ph/0301033 [hep-ph].

[101] J. Gao, M. Guzzi, J. Huston, H.-L. Lai, Z. Li, P. Nadolsky, J. Pumplin, D. Stump, and C. P. Yuan, "CT10 next-to-next-to-leading order global analysis of QCD," Phys. Rev. D89 no. 3, (2014) 033009, arXiv:1302.6246 [hep-ph].

[102] U. Ellwanger, J. Gunion, C. Hugonie, and S. Moretti, "NMSSM Higgs discovery at the LHC," arXiv:hep-ph/0401228 [hep-ph].

[103] R. Benbrik, M. Gomez Bock, S. Heinemeyer, O. Stal, G. Weiglein, et al., "Confronting the MSSM and the NMSSM with the Discovery of a Signal in the two Photon Channel at the LHC," Eur.Phys.J. C72 (2012) 2171, arXiv:1207.1096 [hep-ph].

[104] U. Ellwanger, "A Higgs boson near $125 \mathrm{GeV}$ with enhanced di-photon signal in the NMSSM," JHEP 1203 (2012) 044, arXiv: 1112.3548 [hep-ph].

[105] J.-W. Fan, J.-Q. Tao, Y.-Q. Shen, G.-M. Chen, H.-S. Chen, et al., "Study of diphoton decays of the lightest scalar Higgs boson in the Next-to-Minimal Supersymmetric Standard Model," Chin.Phys. C38 (2014) 073101, arXiv:1309.6394 [hep-ph]. 
[106] J. Cao, Z. Heng, T. Liu, and J. M. Yang, "Di-photon Higgs signal at the LHC: A Comparative study for different supersymmetric models," Phys.Lett. B703 (2011) 462-468, arXiv:1103.0631 [hep-ph].

[107] S. Moretti and S. Munir, "Di-photon Higgs signals at the LHC in the next-to-minimal supersymmetric standard model," Eur.Phys.J. C47 (2006) 791-803, arXiv:hep-ph/0603085 [hep-ph].

[108] M.Guchait,Jacky Kumar in preparation .

[109] A. Altheimer et al., "Jet Substructure at the Tevatron and LHC: New results, new tools, new benchmarks," J. Phys. G39 (2012) 063001, arXiv:1201.0008 [hep-ph].

[110] U. Langenegger, M. Spira, and I. Strebel, "Testing the Higgs Boson Coupling to Gluons," arXiv: 1507.01373 [hep-ph]. 